\title{
Neutrino-Neutrino Scattering and Matter-Enhanced Neutrino Flavor Transformation in Supernovae
}

\author{
Yong-Zhong Qian and George M. Fuller ${ }^{1}$ \\ Institute for Nuclear Theory, HN-12 \\ University of Washington, Seattle, WA 98195
}

\begin{abstract}
We examine matter-enhanced neutrino flavor transformation $\left(\nu_{\tau(\mu)} \rightleftharpoons \nu_{e}\right)$ in the region above the neutrino sphere in Type II supernovae. Our treatment explicitly includes contributions to the neutrino-propagation Hamiltonian from neutrino-neutrino forward scattering. A proper inclusion of these contributions shows that they have a completely negligible effect on the range of $\nu_{e^{-}} \nu_{\tau(\mu)}$ vacuum mass-squared difference, $\delta m^{2}$, and vacuum mixing angle, $\theta$, or equivalently $\sin ^{2} 2 \theta$, required for enhanced supernova shock re-heating. When neutrino background effects are included, we find that $r$-process nucleosynthesis from neutrino-heated supernova ejecta remains a sensitive probe of the mixing between a light $\nu_{e}$ and a $\nu_{\tau(\mu)}$ with a cosmologically significant mass. Neutrino-neutrino scattering contributions are found to have a generally small effect on the $\left(\delta m^{2}, \sin ^{2} 2 \theta\right)$ parameter region probed by $r$-process nucleosynthesis. We point out that the nonlinear effects of the neutrino background extend the range of sensitivity of $r$-process nucleosynthesis to smaller values of $\delta m^{2}$.

PACS numbers: 14.60.Pq, 12.15.Ff, 97.10.Cv, 97.60.Bw
\end{abstract}

${ }^{1}$ Permanent address: Department of Physics, University of California, San Diego, La Jolla, CA 92093-0319. 


\section{Introduction}

In this paper we investigate the problem of matter-enhanced neutrino flavor transformation in the region above the neutrino sphere in Type II supernovae. In particular, we examine the role of contributions to the neutrino-propagation Hamiltonian from neutrinoneutrino forward scattering. A general framework for treating these contributions in the context of the Mikeheyev-Smirnov-Wolfenstein (MSW) neutrino flavor transformation process has been given in Ref. [1] (see Ref. [2] for a numerical study of the case of a pure neutrino gas). Although the role of neutrino-neutrino scattering in the problem of matterenhanced neutrino flavor conversion in supernovae has been treated previously [3, 4], the present paper gives the first complete treatment utilizing the scheme of Ref. [1].

Recent studies have examined MSW tranformation of $\nu_{\tau}$ or $\nu_{\mu}$ into $\nu_{e}$ in the region above the neutrino sphere in the post-core-bounce supernova environment $[5,6]$. These studies suggest that if $\nu_{\tau}$ or $\nu_{\mu}$ has a mass in the cosmologically interesting range of 1-100

$\mathrm{eV}$, then matter-enhanced transformation to $\nu_{e}$ will be possible in this region. Such transformation can result in significant effects on supernova dynamics and/or nucleosynthesis.

If we define, for example, $\left|\nu_{e}\right\rangle$ and $\left|\nu_{\tau}\right\rangle$ to be flavor eigenstates of $\nu_{e}$ and $\nu_{\tau}$, and $\left|\nu_{1}\right\rangle$ and $\left|\nu_{2}\right\rangle$ to be the associated mass eigenstates, then the vacuum mixing angle, $\theta$, is defined through

$$
\begin{aligned}
& \left|\nu_{e}\right\rangle=\cos \theta\left|\nu_{1}\right\rangle+\sin \theta\left|\nu_{2}\right\rangle, \\
& \left|\nu_{\tau}\right\rangle=-\sin \theta\left|\nu_{1}\right\rangle+\cos \theta\left|\nu_{2}\right\rangle .
\end{aligned}
$$


Ref. [5] shows that $\nu_{\tau(\mu)} \rightleftharpoons \nu_{e}$ mixing with $\sin ^{2} 2 \theta \geq 10^{-7}$ in the region above the neutrino sphere at a few hundred milliseconds after the bounce of the core can result in a (30-60)\% increase in the supernova shock energy. Ref. [6] shows that the heavy element nucleosynthesis from the hot bubble region is sensitive to $\nu_{\tau(\mu)} \rightleftharpoons \nu_{e}$ mixing at a level of $\sin ^{2} 2 \theta \sim 10^{-5}$. This hot bubble region forms above the neutrino sphere $\sim 3$ seconds after core bounce. These effects are sensitive to mixing angles far smaller than those which can be probed in laboratory experiments. These supernova effects ultimately may represent our most sensitive probe of putative neutrino dark matter.

However, studies [5] and [6] neglected the off-diagonal contributions of neutrinoneutrino scattering to the flavor-basis neutrino-propagation Hamiltonian. In what follows, we present a detailed study of neutrino flavor transformation in the post-core-bounce supernova environment. Our calculations include all effects of the neutrino background. We have adopted the overall principles and techniques of Ref. [1] in our treatment of neutrinoneutrino and neutrino-electron scattering contributions to the neutrino-propagation Hamiltonian. We find that neutrino background contributions have a negligible effect on the range of $\nu_{e}-\nu_{\tau(\mu)}$ vacuum mass-squared difference, $\delta m^{2}$, and vacuum mixing angle, $\theta$ (or $\sin ^{2} 2 \theta$ ), required for enhanced supernova shock re-heating. A proper treatment of the ensemble average over the neutrino background shows that $r$-process nucleosynthesis from neutrino-heated supernova ejecta remains a sensitive probe of the mixing between a light $\nu_{e}$ and a $\nu_{\tau}\left(\right.$ or $\left.\nu_{\mu}\right)$ with a cosmologically significant mass $\left(m_{\nu_{\tau(\mu)}} \approx 1-100 \mathrm{eV}\right)$.

In Sec. II we discuss a general framework for treating neutrino flavor transformation in the supernova environment. In Sec. III we compute neutrino flavor transformation 
probabilities as functions of $\delta m^{2}$ and $\sin ^{2} 2 \theta$ relevant for the shock re-heating and hot bubble $/ r$-process nucleosynthesis epochs of the supernova. We give conclusions in Sect. IV.

\section{The Neutrino-Propagation Hamiltonian in Supernovae}

The general problem of the time evolution of the full density matrix for an ensemble of three flavors of neutrinos and antineutrinos with an electron/positron background and a nucleon background is a daunting one. Several formal approaches to this problem have been made (cf. Ref. [1] and references therein). In the present paper, we shall only summarize the salient features of this previous work and taylor our subsequent discussion to the particular problem of neutrino propagation and flavor transformation in the region of the supernova environment above the neutrino sphere. Considerable simplification of the problem can be realized in this case.

The general time evolution of the neutrino density matrix $\rho$ can be summarized as

$$
i \dot{\rho}=[H, \rho],
$$

where $\rho=\sum_{i j} \rho_{i j}|i\rangle\langle j|, \dot{\rho}=d \rho / d t$, and $i$ and $j$ refer to all neutrino quantum numbers including momentum (energy), flavor, helicity, charge conjugation eigenvalue, etc. In Eq. (2), $H$ is the full Schrödinger picture Hamiltonian including all neutrino self interactions as well as interactions with the $e^{ \pm}$and nucleon backgrounds.

Without loss of generality we can follow a particular momentum component of Eq. (2) (cf. Ref. [1]), or equivalently, the associated Schrödinger equation for the time evolution 
of neutrino field amplitudes for a given momentum. The Hamiltonian operator in this case would have the dimensionality of the density matrix for the single momentum state (e.g., $12 \times 12$ for three Dirac neutrino flavors, since each neutrino state has either right-handed or left-handed helicity, and is either a neutrino or an antineutrino).

We argue that further simplification of this problem can be made through approximations motivated by the particular distribution functions for $\nu_{e}, \bar{\nu}_{e}, \nu_{\mu}, \bar{\nu}_{\mu}, \nu_{\tau}$, and $\bar{\nu}_{\tau}$ which obtain in the region above the neutrino sphere in the post-core-bounce epoch of Type II supernovae. Since the distribution functions for $\nu_{\mu}, \bar{\nu}_{\mu}, \nu_{\tau}$, and $\bar{\nu}_{\tau}$ are all expected to be essentially identical, mixings between neutrinos in this sector will have no effect on any aspect of supernova physics. In other words, we need only consider mixings between $\nu_{e}$ and either $\nu_{\mu}$ or $\nu_{\tau}$. If, as seems likely, the vacuum mass heirachy for neutrinos satisfies $m_{\nu_{\tau(\mu)}}>m_{\nu_{e}}$, then we need only consider matter-enhanced mixing among neutrinos, as antineutrino mixing is supressed by matter effects.

The masses $m_{\nu_{\tau(\mu)}} \approx 1-100 \mathrm{eV}$ of interest in the post-core-bounce supernova environment are very small compared to the typical neutrino energies (average neutrino energy $\left\langle E_{\nu}\right\rangle$ is about or greater than $\left.10 \mathrm{MeV}\right)$. In this case we can neglect the population of right-handed Dirac neutrinos and left-handed Dirac antineutrinos produced by scattering processes. This is because helicity-flipping rates are proportional to $\left(m_{\nu} / E_{\nu}\right)^{2}$.

Taking advantage of these features allows us to reduce the dimensionality of the Hamiltonian in Eq. (2) to $2 \times 2$ for the Dirac neutrino case. If neutrinos are Majorana particles, then we have only left-handed neutrinos and right-handed antineutrinos, and again the Hamiltonian of interest is $2 \times 2$. 
In any case, the neutrinos of interest in supernovae will be extremely relativistic, so that we can approximate the neutrino energy as $E_{\nu}=\sqrt{p^{2}+m^{2}} \approx p+m^{2} / 2 p$. The first term in this expression, $p$, the momentum, just gives an overall phase to the coherent propagating neutrino state and can be ignored without loss of generality. The second term, $m^{2} / 2 p$, is responsible for the relevant neutrino mixing behavior. The part of the Hamiltonian corresponding to the $m^{2} / 2 p$ term in vacuum, $H_{\mathrm{v}}$, can be written in the flavor basis (e.g., $\left.\left|\nu_{e}\right\rangle,\left|\nu_{\tau}\right\rangle\right)$ as

$$
H_{\mathrm{v}}=\frac{\Delta}{2}\left(\begin{array}{cc}
-\cos 2 \theta & \sin 2 \theta \\
\sin 2 \theta & \cos 2 \theta
\end{array}\right)
$$

where $\theta$ is the vacuum mixing angle as in Eq. (1), $\Delta=\delta m^{2} / 2 E_{\nu}$, and $\delta m^{2} \equiv m_{2}^{2}-m_{1}^{2}$, with $m_{1}$ and $m_{2}$ the vacuum mass eigenvalues corresponding to the mass eigenstates $\left|\nu_{1}\right\rangle$ and $\left|\nu_{2}\right\rangle$, respectively.

In matter the relation between the flavor basis and the mass basis can be written as in Eq. (1), but with the vacuum mixing angle replaced by an appropriate matter mixing angle $\theta_{n}$. For illustrative purposes consider the case where the only contribution to the effective mass difference between neutrino flavors comes from charged-current exchange scattering on electrons. We take the net number density of electrons to be

$$
n_{e} \equiv n_{e^{-}}-n_{e^{+}},
$$

where $n_{e^{-}}\left(n_{e^{+}}\right)$is the total proper number density of negatrons (positrons). The electron fraction $Y_{e}$ is defined in terms of the total baryon rest mass density $\rho$ and Avogardro's number $N_{\mathrm{A}}$ by

$$
n_{e}=\rho Y_{e} N_{\mathrm{A}} .
$$


The contribution to the Hamiltonian from neutrino-electron exchange scattering is

$$
A=\sqrt{2} G_{\mathrm{F}} n_{e},
$$

where $G_{\mathrm{F}}$ is the Fermi constant. In Fig. 1 we show a generic Feynman graph for $\nu_{e^{-}}$ $e$ scattering. To obtain the result in Eq. (5) one must sum graphs for $\nu_{e^{-}}^{-}$and $\nu_{e^{-}}$ $e^{+}$scattering over the appropriate $e^{ \pm}$distribution functions. In this case the neutrinopropagation Hamiltonian, $H_{e}$, can be written as

$$
H_{e}=\frac{\Delta_{\text {eff }}}{2}\left(\begin{array}{cc}
-\cos 2 \theta_{n} & \sin 2 \theta_{n} \\
\sin 2 \theta_{n} & \cos 2 \theta_{n}
\end{array}\right)=\frac{1}{2}\left(\begin{array}{cc}
-\Delta \cos 2 \theta+A & \Delta \sin 2 \theta \\
\Delta \sin 2 \theta & \Delta \cos 2 \theta-A
\end{array}\right)
$$

where $\Delta_{\text {eff }}=\sqrt{(\Delta \cos 2 \theta-A)^{2}+\Delta^{2} \sin ^{2} 2 \theta}$. In these expressions the matter mixing angle, $\theta_{n}$, is related to the vacuum mixing angle $\theta$ and the local net electron number density through

$$
\begin{aligned}
\sin 2 \theta_{n} & =\frac{\Delta \sin 2 \theta}{\sqrt{(\Delta \cos 2 \theta-A)^{2}+\Delta^{2} \sin ^{2} 2 \theta}}, \\
\cos 2 \theta_{n} & =\frac{\Delta \cos 2 \theta-A}{\sqrt{(\Delta \cos 2 \theta-A)^{2}+\Delta^{2} \sin ^{2} 2 \theta}} .
\end{aligned}
$$

The amplitudes for antineutrino-electron $\left(\bar{\nu}_{e^{-}}\right)$exchange scattering and neutrinoelectron $\left(\nu_{e^{-}} e\right)$ exchange scattering have opposite signs. This implies that $\bar{\nu}_{e}-e$ exchange scattering gives a contribution $-A$ to the flavor-basis interaction Hamiltonian for $\bar{\nu}_{e}$. In this case the matter mixing angle for antineutrinos, $\bar{\theta}_{n}$, satisfies

$$
\begin{aligned}
\sin 2 \bar{\theta}_{n} & =\frac{\Delta \sin 2 \theta}{\sqrt{(\Delta \cos 2 \theta+A)^{2}+\Delta^{2} \sin ^{2} 2 \theta}} \\
\cos 2 \bar{\theta}_{n} & =\frac{\Delta \cos 2 \theta+A}{\sqrt{(\Delta \cos 2 \theta+A)^{2}+\Delta^{2} \sin ^{2} 2 \theta}} .
\end{aligned}
$$


We note that the vacuum mixing angles for the neutrino and antineutrino sectors are the same.

It is evident from Eqs. (7a \& b) that matter effects can give enhancement of flavor mixing in the neutrino sector. Mixing is maximal at a mass level crossing, or resonance, where $\Delta \cos 2 \theta=A[7]$. On the other hand, Eqs. (8a \& b) show that matter effects give supression of flavor mixing in the antineutrino sector.

In the supernova environment, however, the neutrino background and the resultant neutrino-neutrino forward exchange-scattering effects necessitate some modification of the above treatment of neutrino flavor transformation. In the region above the neutrino sphere in post-core-bounce Type II supernovae the neutrino fluxes can be sizable (see, for example, the discussion in Ref. [6]). Individual neutrinos emitted from the neutrino sphere can be described as coherent states. However, each emitted neutrino is related to every other emitted neutrino in an incoherent fashion. In other words, these different individual (or single) neutrino states have random relative phases, as is characteristic of a thermal emission process. The total neutrino field is properly a mixed ensemble of individual neutrino states. It is not a coherent many-body state. Accordingly, the total neutrino density matrix is an incoherent sum over each single neutrino density matrix.

For a single neutrino emitted at the neutrino sphere as a $\nu_{\alpha}$ (e.g., in flavor state $\alpha=e, \tau$ for the case of two-neutrino mixing) we can represent its state at some point above the neutrino sphere as

$$
\left|\psi_{\nu_{\alpha}}\right\rangle=a_{1 \alpha}(t)\left|\nu_{1}(t)\right\rangle+a_{2 \alpha}(t)\left|\nu_{2}(t)\right\rangle,
$$


where $\left|\nu_{1}(t)\right\rangle$ and $\left|\nu_{2}(t)\right\rangle$ are the instantaneous physical mass eigenstates of the full neutrino-propagation Hamiltonian, and $a_{1 \alpha}(t)$ and $a_{2 \alpha}(t)$ are the corresponding complex amplitudes. Normalization requires that we take $\left|a_{1 \alpha}(t)\right|^{2}+\left|a_{2 \alpha}(t)\right|^{2}=1$. In these expressions the time, $t$, could be any evolutionary parameter (e.g., density, radius, etc.) along the neutrino's path from its creation position at the neutrino sphere to a point at radius $r$. The single neutrino density matrix is then given by

$$
\begin{aligned}
\left|\psi_{\nu_{\alpha}}\right\rangle\left\langle\psi_{\nu_{\alpha}}\right| & =\left|a_{1 \alpha}(t)\right|^{2}\left|\nu_{1}(t)\right\rangle\left\langle\left.\nu_{1}(t)|+| a_{2 \alpha}(t)\right|^{2} \mid \nu_{2}(t)\right\rangle\left\langle\nu_{2}(t)\right| \\
& +a_{1 \alpha}^{*}(t) a_{2 \alpha}(t)\left|\nu_{2}(t)\right\rangle\left\langle\nu_{1}(t)\left|+a_{1 \alpha}(t) a_{2 \alpha}^{*}(t)\right| \nu_{1}(t)\right\rangle\left\langle\nu_{2}(t)\right| .
\end{aligned}
$$

The density matrix representing the mixed ensemble of single neutrino states all with momentum $\mathbf{p}$ can be written as the incoherent sum

$$
\rho_{\mathbf{p}} d^{3} \mathbf{p}=\sum_{\alpha} d n_{\nu_{\alpha}}\left|\psi_{\nu_{\alpha}}\right\rangle\left\langle\psi_{\nu_{\alpha}}\right|
$$

In this expression the sum runs over, for example, $\alpha=e, \tau$, while $d n_{\nu_{\alpha}}$ is the local differential number density of $\nu_{\alpha}$ neutrinos with momentum $\mathbf{p}$ in interval $d^{3} \mathbf{p}$. The local differential $\nu_{\alpha}$ neutrino number density at a point at radius $r$ above a neutrino sphere with radius $R_{\nu}$ is

$$
d n_{\nu_{\alpha}} \approx n_{\nu_{\alpha}}^{0} f_{\nu_{\alpha}}\left(E_{\nu_{\alpha}}\right) d E_{\nu_{\alpha}}\left(\frac{d \Omega_{\mathbf{p}}}{4 \pi}\right)
$$

where $d \Omega_{\mathbf{p}}$ is the differential solid angle (pencil of directions) along the neutrino momentum $\mathbf{p}\left(|\mathbf{p}| \approx E_{\nu_{\alpha}}\right), n_{\nu_{\alpha}}^{0}$ is the $\nu_{\alpha}$ neutrino number density at the neutrino sphere, and $f_{\nu_{\alpha}}\left(E_{\nu_{\alpha}}\right)$ is the normalized $\nu_{\alpha}$ energy distribution function. We can show [6] that a good approximation for $n_{\nu_{\alpha}}^{0}$ is

$$
n_{\nu_{\alpha}}^{0} \approx \frac{L_{\nu_{\alpha}}}{\left\langle E_{\nu_{\alpha}}\right\rangle} \frac{1}{\pi R_{\nu}^{2} c}
$$


where $L_{\nu_{\alpha}}$ is the luminosity in $\nu_{\alpha}$ neutrinos, $\left\langle E_{\nu_{\alpha}}\right\rangle$ is the average $\nu_{\alpha}$ neutrino energy, and $c$ is the speed of light. The normalized $\nu_{\alpha}$ neutrino energy distribution function can be well approximated by

$$
f_{\nu_{\alpha}}\left(E_{\nu_{\alpha}}\right) \approx \frac{1}{F_{2}(0)} \frac{1}{T_{\nu_{\alpha}}^{3}} \frac{E_{\nu_{\alpha}}^{2}}{\exp \left(E_{\nu_{\alpha}} / T_{\nu_{\alpha}}\right)+1}
$$

where the rank 2 Fermi integral with argument zero is $F_{2}(0) \approx 1.803$, and where $T_{\nu_{\alpha}}$ is the $\nu_{\alpha}$ neutrino sphere temperature. The average $\nu_{\alpha}$ neutrino energy is related to the appropriate neutrino sphere temperature by $\left\langle E_{\nu_{\alpha}}\right\rangle \approx 3.15 T_{\nu_{\alpha}}$.

In the region of the supernova above the neutrino sphere, the range of the solid angle contribution allowed in Eq. (12a) is restricted to be within the solid angle subtended by the neutrino sphere as seen from a point at radius $r$. The geometrical arrangement of a neutrino sphere with radius $R_{\nu}$, a point above the neutrino sphere at radius $r$, and various neutrino paths are depicted in Fig. 2.

We can now write the full flavor-basis neutrino-propagation Hamiltonian as a sum of vacuum mass and electron background contributions, $H_{e}$, and neutrino background contributions, $H_{\nu \nu}$ :

$$
H=H_{e}+H_{\nu \nu}
$$

where $H_{\nu \nu}$ represents the ensemble average over neutrino-neutrino interactions using the density matrix in Eq. (11). For a neutrino with energy $E_{\nu}$ and momentum p propagating radially outside the neutrino sphere we can write

$$
H_{\nu \nu}=\sqrt{2} G_{\mathrm{F}} \int\left(1-\cos \theta_{\mathbf{q}}\right)\left(\rho_{\mathbf{q}}-\bar{\rho}_{\mathbf{q}}\right) d^{3} \mathbf{q},
$$


where $\bar{\rho}_{\mathbf{q}}$ is the density matrix for antineutrinos with momentum $\mathbf{q}$ (defined in obvious analogy to $\rho_{\mathbf{q}}$ in Eq. [11]) and $\theta_{\mathbf{q}}$ is the angle between the direction of the propagating neutrino with momentum $\mathbf{p}$ and the directions of other neutrinos in the ensemble with momentum q. We can generalize the expression for $H_{\nu \nu}$ in Eq. (13b) for non-radially propagating neutrinos by replacing $\cos \theta_{\mathbf{q}}$ with $\mathbf{q} \cdot \mathbf{p} /\{|\mathbf{q}||\mathbf{p}|\}$.

It is convenient to recast Eq. (13b) in the form

$$
H_{\nu \nu}=\frac{1}{2}\left(\begin{array}{cc}
B & B_{e \tau} \\
B_{\tau e} & -B
\end{array}\right)+\frac{\sqrt{2}}{2} G_{\mathrm{F}} \int\left(1-\cos \theta_{\mathbf{q}}\right) \operatorname{Tr}\left(\rho_{\mathbf{q}}-\bar{\rho}_{\mathbf{q}}\right) d^{3} \mathbf{q}
$$

Note that the second term in this equation is simply proportional to the identity matrix, implying that it provides only an overall phase in the propagating neutrino state and can be ignored.

In the first term in Eq. (14) there are two contributions to the neutrino-propagation Hamiltonian, $B$ and $B_{e \tau}\left(B_{\tau e}\right)$, where

$$
\begin{aligned}
B & =\sqrt{2} G_{\mathrm{F}} \int\left(1-\cos \theta_{\mathbf{q}}\right)\left\{\left(\rho_{\mathbf{q}}-\bar{\rho}_{\mathbf{q}}\right)_{e e}-\left(\rho_{\mathbf{q}}-\bar{\rho}_{\mathbf{q}}\right)_{\tau \tau}\right\} d^{3} \mathbf{q}, \\
B_{e \tau} & =2 \sqrt{2} G_{\mathrm{F}} \int\left(1-\cos \theta_{\mathbf{q}}\right)\left(\rho_{\mathbf{q}}-\bar{\rho}_{\mathbf{q}}\right)_{e \tau} d^{3} \mathbf{q}, \\
B_{\tau e} & =2 \sqrt{2} G_{\mathrm{F}} \int\left(1-\cos \theta_{\mathbf{q}}\right)\left(\rho_{\mathbf{q}}-\bar{\rho}_{\mathbf{q}}\right)_{\tau e} d^{3} \mathbf{q},
\end{aligned}
$$

where, for example, by $\left(\rho_{\mathbf{q}}\right)_{e \tau}$ we mean the matrix element of the density matrix operator, $\left\langle\nu_{e}\left|\rho_{\mathbf{q}}\right| \nu_{\tau}\right\rangle$, while by $\left(\bar{\rho}_{\mathbf{q}}\right)_{e \tau}$ we mean $\left\langle\bar{\nu}_{e}\left|\rho_{\mathbf{q}}\right| \bar{\nu}_{\tau}\right\rangle$.

Here $B$ corresponds to the forward neutrino-neutrino exchange-scattering contributions to the neutrino effective mass. These contributions are the analogs of the $\nu_{e}-e$ exchange-scattering term, $A$, in Eqs. (5) and (6). Generic Feynman graphs for these neutrino-neutrino exchange processes are shown in Fig. 3a for $\nu_{e}-\nu_{e}$ scattering and in Fig. 
3b for $\nu_{\tau}-\nu_{\tau}$ scattering. We will later refer to $B$ as the "diagonal" contribution of the neutrino background to the flavor-basis neutrino-propagation Hamiltonian.

The neutrino background also provides "off-diagonal" terms in the flavor-basis neutrino-propagation Hamiltonian. These are, for example, the $B_{e \tau}$ and $B_{\tau e}$ terms above. They arise because the background neutrinos are not in flavor eigenstates [1]. We show graphically these contributions for $\nu_{e}$ and $\nu_{\tau}$ neutrinos with momenta $\mathbf{p}$ and $\mathbf{q}$ in Fig. 4. The corresponding diagonal and off-diagonal contributions to the flavor-basis antineutrinopropagation Hamiltonian from the neutrino background are $-B$ and $-B_{e \tau}\left(-B_{\tau e}\right)$, respectively.

Considerable simplification in the evaluation of $B, B_{e \tau}\left(B_{\tau e}\right)$ can be realized by adroit attention to the phases in the expression for the single neutrino density matrix in Eq. (10). Note that the last two terms in Eq. (10) are cross terms. They have coefficients $a_{1}^{*}(t) a_{2}(t)$ and $a_{1}(t) a_{2}^{*}(t)$, respectively. Each cross term is proportional to a factor $\sim \exp \left[i \int \omega_{12}(t) d t\right]$, with $\omega_{12}$ the difference in the local neutrino flavor-oscillation frequencies of the two mass eigenstates $\left|\nu_{1}(t)\right\rangle$ and $\left|\nu_{2}(t)\right\rangle$. These oscillation frequencies are, in turn, dependent on the local density.

In both the early post-core-bounce shock re-heating epoch (time post-core-bounce $\left.t_{\mathrm{PB}} \sim 0.1-1 \mathrm{~s}\right)$ and in the hot bubble/r-process nucleosynthesis epoch $\left(t_{\mathrm{PB}} \sim 3-15 \mathrm{~s}\right)$ the electron number density predominantly determines the neutrino flavor-oscillation frequency in the region just above the neutrino sphere $[5,6]$. This is because the net neutrino number densities are negligible compared to the electron number densities $\left(n_{e} \sim 10^{35} \mathrm{~cm}^{-3}\right)$ in this region $[5,6]$. The Hamiltonian $H_{e}$ in Eq. (6) is by itself sufficient to determine the 
neutrino flavor-oscillation frequencies in this region. The local neutrino flavor-oscillation frequency difference in this case is $\omega_{12} \approx \Delta_{\text {eff }}=\sqrt{(\Delta \cos 2 \theta-A)^{2}+\Delta^{2} \sin ^{2} 2 \theta}$.

Furthermore, for the cosmologically interesting range $\delta m^{2}=1-100 \mathrm{eV}^{2}$, the electron number density near the neutrino sphere greatly exceeds the MSW resonance densities for neutrinos with energies $E_{\nu} \sim 10 \mathrm{MeV}$. In this case the neutrino paths shown in Fig. 2 will always cross a region where the electron number densities dominate the neutrino number densities prior to entering the resonance region. Therefore the local neutrino flavor-oscillation frequency difference will be $\omega_{12} \approx \Delta_{\text {eff }} \approx A=\sqrt{2} G_{\mathrm{F}} n_{e}$ along some part of every neutrino's path.

When taking the ensemble average over the neutrino background we necessarily integrate $\rho_{\mathbf{p}}$ over neutrino momentum directions to a point at radius $r$. We thereby also average over the oscillating cross terms in Eq. (10). In addition, neutrinos with different momentum directions travel on paths with different lengths to arrive at a point at radius $r$. These different path lengths then give rise to different phases for the oscillating factor $\sim \exp \left[i \int \omega_{12}(t) d t\right]$ in the cross terms in Eq. (10). In fact it is clear from Fig. 2 that each neutrino path from the neutrino sphere to a point at radius $r$ will have a path length which depends on the polar angle. For neutrinos with momentum magnitude $|\mathbf{p}|$ each path with a different polar angle will have a different phase entering into the cross term coefficients of Eq. (10).

The phase difference $\delta \phi$ acquired in going through a region of electron number density $n_{e}$ with a difference in path length $\delta r$ is then

$$
\delta \phi \approx \sqrt{2} G_{\mathrm{F}} n_{e} \delta r \approx 642\left(\frac{n_{e}}{10^{35} \mathrm{~cm}^{-3}}\right)\left(\frac{\delta r}{1 \mathrm{~cm}}\right)
$$


Path length differences of order $1 \mathrm{~cm}$ give rise to phase differences of $\gg 2 \pi$ ! It is obvious that the cross terms in the single neutrino density matrix in Eq. (10) vanish when averaged over all neutrino momentum directions. This argument can also be applied to the evaluation of $\bar{\rho}_{\mathbf{p}}$ since $\omega_{12}>\sqrt{2} G_{\mathrm{F}} n_{e}$ everywhere for antineutrinos.

Clearly, we need only consider the first two terms of Eq. (10) in evaluating matrix elements of the density matrices $\rho_{\mathbf{p}}$ and $\bar{\rho}_{\mathbf{p}}$ (cf. Eq. [11]). This will allow considerable simplification in computation of $B$ and $B_{e \tau}\left(B_{\tau e}\right)$ from Eqs. $(15 \mathrm{a}-\mathrm{c})$.

Failure to properly perform the angular part of the ensemble average would result in the retention of non-zero cross terms in the neutrino density matrix elements, Eqs. (15ac). This would introduce a spurious, and unphysical, "coherence" in the treatment of the neutrino background. In fact, the angular part of the ensemble average over the neutrino background is a key point in determining neutrino flavor evolution in the region above the neutrino sphere in supernovae.

Note that $B_{e \tau}=B_{\tau e}$ since the terms in the ensemble averages, Eqs. $(15 \mathrm{a}-\mathrm{c})$, are all real and the Hamiltonian must be Hermitian. The terms in Eqs. (15a-c) are all real because of the vanishing of the cross terms in the momentum direction average over the single neutrino density matrices. The full flavor-basis Hamiltonian which includes both the electron and neutrino backgrounds is now

$$
H=H_{e}+H_{\nu \nu}=\frac{1}{2}\left(\begin{array}{cc}
-\Delta \cos 2 \theta+A+B & \Delta \sin 2 \theta+B_{e \tau} \\
\Delta \sin 2 \theta+B_{e \tau} & \Delta \cos 2 \theta-A-B
\end{array}\right) .
$$

In analogy to the discussion preceding Eq. (6) we can rewrite this Hamiltonian as

$$
H=\frac{\Delta_{\mathrm{H}}}{2}\left(\begin{array}{cc}
-\cos 2 \theta_{\mathrm{H}} & \sin 2 \theta_{\mathrm{H}} \\
\sin 2 \theta_{\mathrm{H}} & \cos 2 \theta_{\mathrm{H}}
\end{array}\right) .
$$


In this expression we have defined a full effective mixing angle, $\theta_{\mathrm{H}}$, which, in analogy to Eq. (1), gives the relations between the flavor basis and the instantaneous mass basis including the effects of both the electron and neutrino backgrounds:

$$
\begin{aligned}
& \left|\nu_{e}(t)\right\rangle=\cos \theta_{\mathrm{H}}(t)\left|\nu_{1}(t)\right\rangle+\sin \theta_{\mathrm{H}}(t)\left|\nu_{2}(t)\right\rangle, \\
& \left|\nu_{\tau}(t)\right\rangle=-\sin \theta_{\mathrm{H}}(t)\left|\nu_{1}(t)\right\rangle+\cos \theta_{\mathrm{H}}(t)\left|\nu_{2}(t)\right\rangle .
\end{aligned}
$$

We have defined $\Delta_{\mathrm{H}}$ as

$$
\Delta_{\mathrm{H}} \equiv \sqrt{(\Delta \cos 2 \theta-A-B)^{2}+\left(\Delta \sin 2 \theta+B_{e \tau}\right)^{2}} .
$$

The full effective mixing angle satisfies

$$
\begin{aligned}
\sin 2 \theta_{\mathrm{H}} & =\frac{\Delta \sin 2 \theta_{\mathrm{H}}+B_{e \tau}}{\sqrt{(\Delta \cos 2 \theta-A-B)^{2}+\left(\Delta \sin 2 \theta+B_{e \tau}\right)^{2}}}, \\
\cos 2 \theta_{\mathrm{H}} & =\frac{\Delta \cos 2 \theta_{\mathrm{H}}-A-B}{\sqrt{(\Delta \cos 2 \theta-A-B)^{2}+\left(\Delta \sin 2 \theta+B_{e \tau}\right)^{2}}} .
\end{aligned}
$$

Note that in the absence of a neutrino background $\Delta_{\mathrm{H}}=\Delta_{\text {eff }}$ and $\theta_{\mathrm{H}}=\theta_{n}$. The corresponding expressions for the full effective mixing angle, $\bar{\theta}_{\mathrm{H}}$, in the antineutrino sector are obtained by replacing $A, B$, and $B_{e \tau}$ with $-A,-B$, and $-B_{e \tau}$, respectively.

Since the cross terms in the single neutrino density matrix will give no contribution to the ensemble average, we can write a reduced expression for the single neutrino density matrix in terms of flavor-basis eigenbras and eigenkets:

$$
\begin{aligned}
\left(\left|\psi_{\nu_{\alpha}}\right\rangle\left\langle\psi_{\nu_{\alpha}}\right|\right)_{\text {reduced }} & =\left\{\frac{1}{2}-\left[\frac{1}{2}-\left|a_{1 \alpha}(t)\right|^{2}\right] \cos 2 \theta_{\mathrm{H}}(t)\right\}\left|\nu_{e}\right\rangle\left\langle\nu_{e}\right| \\
& +\left\{\frac{1}{2}-\left[\frac{1}{2}+\left|a_{1 \alpha}(t)\right|^{2}\right] \cos 2 \theta_{\mathrm{H}}(t)\right\}\left|\nu_{\tau}\right\rangle\left\langle\nu_{\tau}\right| \\
& +\left[\frac{1}{2}-\left|a_{1 \alpha}(t)\right|^{2}\right] \sin 2 \theta_{\mathrm{H}}(t)\left(\left|\nu_{e}\right\rangle\left\langle\nu_{\tau}|+| \nu_{\tau}\right\rangle\left\langle\nu_{e}\right|\right)
\end{aligned}
$$


With this form for the single neutrino density matrix, it is straightforward to evaluate flavor-basis matrix elements of the density matrix operator. For example, the expressions in Eqs. $(15 \mathrm{a}-\mathrm{c})$ become

$$
\begin{array}{r}
B=-\sqrt{2} G_{\mathrm{F}} \sum_{\alpha} \int\left(1-\cos \theta_{\mathbf{q}}\right)\left\{\left[1-2\left|a_{1 \alpha}(t)\right|^{2}\right] \cos 2 \theta_{\mathrm{H}}(t) d n_{\nu_{\alpha}}\right. \\
\left.-\left[1-2\left|\bar{a}_{1 \alpha}(t)\right|^{2}\right] \cos 2 \bar{\theta}_{\mathrm{H}}(t) d n_{\bar{\nu}_{\alpha}}\right\}, \\
B_{e \tau}=\sqrt{2} G_{\mathrm{F}} \sum_{\alpha} \int\left(1-\cos \theta_{\mathbf{q}}\right)\left\{\left[1-2\left|a_{1 \alpha}(t)\right|^{2}\right] \sin 2 \theta_{\mathrm{H}}(t) d n_{\nu_{\alpha}}\right. \\
\left.-\left[1-2\left|\bar{a}_{1 \alpha}(t)\right|^{2}\right] \sin 2 \bar{\theta}_{\mathrm{H}}(t) d n_{\bar{\nu}_{\alpha}}\right\} .
\end{array}
$$

In these expressions $a_{1 \alpha}(t)$ is the amplitude to be in the instantaneous mass eigenstate $\left|\nu_{1}(t)\right\rangle$ for an individual neutrino of momentum $\mathbf{q}$ which was created at the neutrino sphere $(t=0)$ in flavor eigenstate $\left|\nu_{\alpha}\right\rangle$. Likewise, $\bar{a}_{1 \alpha}(t)$ is the amplitude to be in the instantaneous

mass eigenstate $\left|\bar{\nu}_{1}(t)\right\rangle$ for an antineutrino of momentum q created at the neutrino sphere in flavor eigenstate $\left|\bar{\nu}_{\alpha}\right\rangle$. The expressions $d n_{\nu_{\alpha}}$ and $d n_{\bar{\nu}_{\alpha}}$ are as given in Eq. (12a), e.g., $d n_{\nu_{\alpha}} \approx n_{\nu_{\alpha}}^{0} f_{\nu_{\alpha}}\left(E_{\nu_{\alpha}}\right) d E_{\nu_{\alpha}}\left(d \Omega_{\mathbf{q}} / 4 \pi\right)$.

It remains to evaluate these expressions for the particular conditions (electron density run and neutrino distribution functions) which obtain for the shock re-heating and hot bubble $/ r$-process nucleosynthesis epochs.

\section{Neutrino Flavor Transformation in the Supernova Environment}

In this section we examine neutrino flavor transformation in the region above the neutrino sphere in models of post-core-bounce Type II supernovae. There are several aspects 
of the problem of neutrino flavor transformation in supernovae which are significantly different from conventional computations of MSW flavor conversion in the sun. Foremost among these is the necessity of treating the neutrino background. In addition, the geometry of neutrino emission from a neutrino sphere in a supernova is quite different from the solar case, where the neutrino source is distributed throughout the core.

Bearing these points in mind, we can formally transform the full flavor-basis Hamiltonian in Eqs. (17a \& b) to the basis of the instantaneous mass eigenstates $\left|\nu_{1}(t)\right\rangle$ and $\left|\nu_{2}(t)\right\rangle$. The Schrödinger equation for the time evolution of the amplitudes $a_{1 \alpha}(t)$ and $a_{2 \alpha}(t)$ (see Eq. [9]) in this basis is then,

$$
i\left(\begin{array}{c}
\dot{a}_{1 \alpha}(t) \\
\dot{a}_{2 \alpha}(t)
\end{array}\right)=\left(\begin{array}{cc}
-\Delta_{\mathrm{H}}(t) / 2 & -i \dot{\theta}_{\mathrm{H}}(t) \\
i \dot{\theta}_{\mathrm{H}}(t) & \Delta_{\mathrm{H}}(t) / 2
\end{array}\right)\left(\begin{array}{c}
a_{1 \alpha}(t) \\
a_{2 \alpha}(t)
\end{array}\right),
$$

where $\dot{a}_{1 \alpha}(t)=d a_{1 \alpha}(t) / d t, \dot{a}_{2 \alpha}(t)=d a_{2 \alpha}(t) / d t$, and $\dot{\theta}_{\mathrm{H}}(t)=d \theta_{\mathrm{H}}(t) / d t$. In this expression we follow the treatment of neutrino propagation and flavor transformation in Ref. [8]. Eq. (23) represents a set of nonlinear first order differential equations for the amplitudes $a_{1 \alpha}(t)$ and $a_{2 \alpha}(t)$. The nonlinearity arises since, in general, $\Delta_{\mathrm{H}}$ and the full effective mixing angle $\theta_{\mathrm{H}}$ each depend on the neutrino background contributions $B$ and $B_{e \tau}$ (Eqs. [19], [20a \& b]). In turn, $B$ and $B_{e \tau}$ depend on the amplitudes $a_{1 \alpha}(t)$ as in Eqs. (22a \& b).

The time evolution of the full effective mixing angle can be found from Eqs. (20a \& b) to be

$$
\dot{\theta}_{\mathrm{H}}(t)=\frac{\dot{B}_{e \tau}(\Delta \cos 2 \theta-A-B)+\left(\Delta \sin 2 \theta+B_{e \tau}\right)(\dot{A}+\dot{B})}{2\left[(\Delta \cos 2 \theta-A-B)^{2}+\left(\Delta \sin 2 \theta+B_{e \tau}\right)^{2}\right]},
$$

where $\dot{A}=d A / d t, \dot{B}=d B / d t$, and $\dot{B}_{e \tau}=d B_{e \tau} / d t$. 
We can define an "adiabaticity parameter" $\gamma(t)$ :

$$
\gamma(t) \equiv \frac{\Delta_{\mathrm{H}}(t)}{2\left|\dot{\theta}_{\mathrm{H}}(t)\right|}
$$

Clearly, the neutrino mass eigenstate evolution is well approximated as being adiabatic when $\gamma(t) \gg 1$. Of course, if $\dot{\theta}_{\mathrm{H}}=0$, the neutrino mass eigenstate evolution is completely adiabatic, as can be seen directly from Eq. (23).

The adiabaticity parameter generally satisfies $\gamma(t) \gg 1$ well away from resonance regions (neutrino mass-level-crossing regions). However, neutrino flavor conversion probabilities depend crucially on $\gamma(t)$ at resonance. We shall denote the value of the adiabaticity parameter at resonance as $\gamma\left(t_{\text {res }}\right)$. Resonance occurs when

$$
\Delta \cos 2 \theta=A+B
$$

We denote the position of this level-crossing point, or resonance, by $t_{\mathrm{res}}$. At resonance,

$$
\gamma\left(t_{\mathrm{res}}\right)=\frac{\left(\Delta \sin 2 \theta+B_{e \tau}\right)^{2}}{|\dot{A}+\dot{B}|}=\frac{\left(\Delta \sin 2 \theta+B_{e \tau}\right)^{2}}{\Delta \cos 2 \theta}\left|\frac{d \ln (A+B)}{d t}\right|_{t_{\mathrm{res}}}^{-1} .
$$

The Landau-Zener probability for the neutrino to jump from one mass eigenstate to the other in the course of transversing a resonance region is [8],

$$
P_{\mathrm{LZ}} \approx \exp \left[-\frac{\pi}{2} \gamma\left(t_{\mathrm{res}}\right)\right]
$$

Unlike the case for solar neutrinos, this expression is always sufficient for calculating neutrino flavor transformation in supernovae [5, 6]. The Landau-Zener formula Eq. (28) is inapplicable for solar neutrino flavor conversion when, for example, neutrinos are created close to their resonance positions. This never occurs in supernovae, where neutrinos originate on the neutrino sphere. The neutrino sphere is always well away from the resonance 
region for the cases we will consider. In addition, solar neutrinos can experience double level crossings when they are created at densities below their resonance density. This does not occur in the post-core-bounce supernova environment.

The very small vacuum mixing angles we shall consider for neutrino flavor conversion in supernovae imply narrow resonance regions. Narrow resonance regions, together with the generally large density scale heights $(0.5-50 \mathrm{~km})$ characterisitic of the region above the neutrino sphere $[5,6]$, imply that the first order Landau-Zener jump probability expression in Eq. (28) is always adequate [5,6]. By first order jump probability we mean that we approximate the density profile as linear across the resonance region.

It is obvious in Eqs. (24-27) that we recover the pure electron-driven neutrino flavor conversion case when the neutrino background disappears (i.e., $B$ and $B_{e \tau}$ vanish everywhere). The neutrino background influences neutrino flavor evolution through resonances in two ways.

First, the diagonal contribution of the neutrino background, $B$, essentially shifts the position of the resonance from the case where only the electron contribution, $A$, is present. This is evident from Eq. (26). The diagonal contribution of the neutrino background also alters the density scale height of weak interaction scattering targets at resonance. The density scale height of weakly interacting targets $\left(|d \ln n / d r|^{-1}\right.$ following Eq. [7] in Ref. [6]) is the $|d \ln (A+B) / d t|^{-1}$ term in Eq. (27).

The off-diagonal contribution of the neutrino background, $B_{e \tau}$, has the effect of altering the adiabaticity of the neutrino flavor evolution at resonance. This is clear from Eq. (27), where $B_{e \tau}$ appears in the expression for $\gamma\left(t_{\mathrm{res}}\right)$. If $\Delta \sin 2 \theta \gg\left|B_{e \tau}\right|$ then the 
off-diagonal neutrino background contribution will have little influence on the adiabaticity of neutrino flavor evolution.

However, the diagonal and off-diagonal contributions of the neutrino background influence neutrino flavor evolution in a nonlinear manner, as outlined above. Not only are $B$ and $B_{e \tau}$ determined by the local neutrino distribution functions, but the local neutrino distribution functions are also dependent, in general, on the detailed history of neutrino flavor transformation.

The crux of the problem of treating the nonlinear effects of the neutrino background is the computation of $B$ and $B_{e \tau}$ for the particular local neutrino distribution functions which obtain in the supernova environment. This will be evident if we discuss a simple iterative procedure for computing neutrino flavor transformation at resonance in the presence of a neutrino background.

We can employ the Landau-Zener transformation probability in Eq. (28) to estimate the neutrino flavor conversion probability for a neutrino propagating through a resonance with the following simple procedure. We choose a vacuum mass-squared difference $\delta m^{2}$ and a vacuum mixing angle $\theta$ (equivalently, $\sin ^{2} 2 \theta$ ) for a propagating neutrino of energy $E_{\mathrm{R}}$.

(1.) To begin with, we assume that $B_{e \tau}=0$. We use $\delta m^{2}$ and $\sin ^{2} 2 \theta$, along with $B_{e \tau}=0$, in Eqs. $(20 \mathrm{a} \& \mathrm{~b})$ to get a zero-order estimate for $\cos 2 \theta_{\mathrm{H}}, \sin 2 \theta_{\mathrm{H}}, \cos 2 \bar{\theta}_{\mathrm{H}}$, and $\sin 2 \bar{\theta}_{\mathrm{H}}$. Note that the value of $A$ and $B$ which enter into the expressions for $\cos 2 \theta_{\mathrm{H}}$, $\sin 2 \theta_{\mathrm{H}}, \cos 2 \bar{\theta}_{\mathrm{H}}$, and $\sin 2 \bar{\theta}_{\mathrm{H}}$ are their values at the resonance position, $A\left(t_{\mathrm{res}}\right)$ and $B\left(t_{\mathrm{res}}\right)$. In this case we can replace $A+B$ by $\left(\delta m^{2} / 2 E_{\mathrm{R}}\right) \cos 2 \theta$ wherever it occurs. Eqs. (20a \& 
b) with $B_{e \tau}=0$ can then be written as:

$$
\begin{aligned}
& \sin 2 \theta_{\mathrm{H}}=\frac{\tan 2 \theta}{\sqrt{\left(1-E / E_{\mathrm{R}}\right)^{2}+\tan ^{2} 2 \theta}}, \\
& \cos 2 \theta_{\mathrm{H}}=\frac{1-E / E_{\mathrm{R}}}{\sqrt{\left(1-E / E_{\mathrm{R}}\right)^{2}+\tan ^{2} 2 \theta}}, \\
& \sin 2 \bar{\theta}_{\mathrm{H}}=\frac{\tan 2 \theta}{\sqrt{\left(1+E / E_{\mathrm{R}}\right)^{2}+\tan ^{2} 2 \theta}}, \\
& \cos 2 \bar{\theta}_{\mathrm{H}}=\frac{1+E / E_{\mathrm{R}}}{\sqrt{\left(1+E / E_{\mathrm{R}}\right)^{2}+\tan ^{2} 2 \theta}} .
\end{aligned}
$$

(2.) We employ these approximations for the full effective mixing angle to obtain estimates for $B$ in Eq. (22a).

(3.) So far we have not specified the resonance position. We now use $A$ and the estimate of $B$ from Step (2) to estimate the resonance position through $\left(\delta m^{2} / 2 E_{\mathrm{R}}\right) \cos 2 \theta=$ $A+B$. Note that $A$ and $B$ are position dependent.

(4.) With the resonance position from Step (3) we use Eq. (22b) to estimate $B_{e \tau}$.

(5.) With this estimate for $B_{e \tau}$ we now can re-estimate the full effective mixing angle using

$$
\begin{aligned}
\sin 2 \theta_{\mathrm{H}} & =\frac{\left(\delta m^{2} / 2 E_{\nu}\right) \sin 2 \theta+B_{e \tau}}{\sqrt{\left[\left(\delta m^{2} / 2 E_{\nu}\right)-\left(\delta m^{2} / 2 E_{\mathrm{R}}\right)\right]^{2} \cos ^{2} 2 \theta+\left[\left(\delta m^{2} / 2 E_{\nu}\right) \sin 2 \theta+B_{e \tau}\right]^{2}}}, \\
\cos 2 \theta_{\mathrm{H}} & =\frac{\left[\left(\delta m^{2} / 2 E_{\nu}\right)-\left(\delta m^{2} / 2 E_{\mathrm{R}}\right)\right] \cos 2 \theta}{\sqrt{\left[\left(\delta m^{2} / 2 E_{\nu}\right)-\left(\delta m^{2} / 2 E_{\mathrm{R}}\right)\right]^{2} \cos ^{2} 2 \theta+\left[\left(\delta m^{2} / 2 E_{\nu}\right) \sin 2 \theta+B_{e \tau}\right]^{2}}}, \\
\sin 2 \bar{\theta}_{\mathrm{H}} & =\frac{\left(\delta m^{2} / 2 E_{\nu}\right) \sin 2 \theta-B_{e \tau}}{\sqrt{\left[\left(\delta m^{2} / 2 E_{\nu}\right)+\left(\delta m^{2} / 2 E_{\mathrm{R}}\right)\right]^{2} \cos ^{2} 2 \theta+\left[\left(\delta m^{2} / 2 E_{\nu}\right) \sin 2 \theta-B_{e \tau}\right]^{2}}} \\
\cos 2 \bar{\theta}_{\mathrm{H}} & =\frac{\left[\left(\delta m^{2} / 2 E_{\nu}\right)+\left(\delta m^{2} / 2 E_{\mathrm{R}}\right)\right] \cos 2 \theta}{\sqrt{\left[\left(\delta m^{2} / 2 E_{\nu}\right)+\left(\delta m^{2} / 2 E_{\mathrm{R}}\right)\right]^{2} \cos ^{2} 2 \theta+\left[\left(\delta m^{2} / 2 E_{\nu}\right) \sin 2 \theta-B_{e \tau}\right]^{2}}}
\end{aligned}
$$

(6.) We iterate by returning to Step (2) and re-evaluating $B$.

This procedure must be continued until $B, B_{e \tau}, \theta_{\mathrm{H}}$ and the resonance position $\left(t_{\text {res }}\right)$ converge. Because of the dependence of $B$ and $B_{e \tau}$ on the flavor evolution histories of 
all neutrinos in the ensemble, convergence of this procedure is, in general, problematic. However, if neutrino flavor evolution is adiabatic then the complication of prior histories is eliminated and the above procedure converges rapidly for the conditions which obtain in the region above the neutrino sphere in Type II supernovae. For nonadiabatic neutrino flavor evolution the above procedure, though more laborious, still gives good estimates of the effects of the neutrino background. We shall begin by discussing the case of adiabatic neutrino flavor evolution.

\section{IIIa.) Adiabatic Neutrino Flavor Evolution}

Consider the flavor evolution of antineutrinos. It is generally true everywhere above the neutrino sphere that the contributions of the electrons and neutrinos satisfy $A+B>0$. This is true because $n_{e}$ is everywhere greater than the net neutrino number densities for any neutrino flavor $[5,6]$. For an antineutrino emitted from the neutrino sphere in the $\left|\bar{\nu}_{e}\right\rangle$ flavor eigenstate, it is evident that $\left|\bar{a}_{1 e}(t)\right|^{2} \approx 1$ and $\left|\bar{a}_{1 \tau}(t)\right|^{2} \approx 0$ for all $t$. The effective mass-squared difference for two antineutrino mass eigenstates always increases with density and there is no mass level crossing. The adiabatic approximation for the evolution of the antineutrino mass eigenstates is always good.

The situation is more complicated for neutrinos. However, the approximation of adiabatic evolution of the neutrino mass eigenstates is a particularly simple case to treat in the supernova. A neutrino created in a flavor eigenstate $\left|\nu_{\alpha}\right\rangle$ at the neutrino sphere is very nearly in a mass eigenstate because of the large electron number density there. Subsequent adiabatic evolution then implies that, for example, $\left|a_{1 e}(t)\right|^{2}=0$ and $\left|a_{1 \tau}(t)\right|^{2}=1$ for all 
$t$ (likewise, $\left|a_{2 e}(t)\right|^{2}=1$ and $\left|a_{2 \tau}(t)\right|^{2}=0$ for all $t$ ). In this case the expressions for the neutrino background contributions, Eqs. (22a \& b), become

$$
\begin{aligned}
& B \approx-\sqrt{2} G_{\mathrm{F}} \int\left(1-\cos \theta_{\mathbf{q}}\right)\left[\cos 2 \theta_{\mathrm{H}}(t)\left(d n_{\nu_{e}}-d n_{\nu_{\tau}}\right)+\cos 2 \bar{\theta}_{\mathrm{H}}(t)\left(d n_{\bar{\nu}_{e}}-d n_{\bar{\nu}_{\tau}}\right)\right] \\
& B_{e \tau} \approx \sqrt{2} G_{\mathrm{F}} \int\left(1-\cos \theta_{\mathbf{q}}\right)\left[\sin 2 \theta_{\mathrm{H}}(t)\left(d n_{\nu_{e}}-d n_{\nu_{\tau}}\right)+\sin 2 \bar{\theta}_{\mathrm{H}}(t)\left(d n_{\bar{\nu}_{e}}-d n_{\bar{\nu}_{\tau}}\right)\right] .
\end{aligned}
$$

The evaluation of Eqs. (31a \& b) for particular neutrino distribution functions is straightforward so long as the adiabatic approximation obtains. To begin with, consider the computation of $B$ from Eq. (31a) in the limit where $B_{e \tau}=0$. The result so obtained will be valid if we can later show that $\left|B_{e \tau}\right| \ll\left(\delta m^{2} / 2 E_{\mathrm{R}}\right) \sin 2 \theta$.

With the approximation that $B_{e \tau}$ is small the integrals over the neutrino distribution functions $d n_{\nu_{e}}, d n_{\nu_{\tau}}, d n_{\bar{\nu}_{e}}$, and $d n_{\bar{\nu}_{\tau}}$ can be separated into an angular part and an energy part. This is due to the fact that when $B_{e \tau}$ is small $\theta_{\mathrm{H}}$ and $\bar{\theta}_{\mathrm{H}}$ essentially become functions of energy alone. For a radially propagating neutrino, the angular part of the integral in Eq. (31a) then becomes,

$$
\int\left(1-\cos \theta_{\mathbf{q}}\right) \frac{d \Omega_{\mathbf{q}}}{4 \pi}=\frac{1}{2} \int_{0}^{\theta_{0}}(1-\cos \theta) \sin \theta d \theta=\frac{1}{4}\left[1-\sqrt{1-\left(R_{\nu} / r\right)^{2}}\right]^{2} .
$$

In this equation $r$ is the radius of the point at which we evaluate $B$ and $\theta_{0}$ is the polar angle of the limb of the neutrino sphere as seen from this point. Frequently we are interested in regions sufficiently distant from the neutrino sphere that we can take $r \gg R_{\nu}$. In this limit, the radial neutrino path to the point at radius $r$ is a good representation of all neutrino paths to that point, and we can approximate

$$
\int\left(1-\cos \theta_{\mathbf{q}}\right) \frac{d \Omega_{\mathbf{q}}}{4 \pi} \approx \frac{1}{16} \frac{R_{\nu}^{4}}{r^{4}}
$$


It is obvious from this expression that the diagonal contribution of the neutrino background is sensitive to position.

The integration of the remaining energy dependent terms in Eq. (31a) is simple if we employ the approximate energy spectra in Eq. (12c). The energy part of Eq. (31a) is then,

$$
\begin{aligned}
& \int \cos 2 \theta_{\mathrm{H}} f_{\nu_{\alpha}}\left(E_{\nu_{\alpha}}\right) d E_{\nu_{\alpha}} \approx F_{\nu}\left(\theta, E_{\mathrm{R}} / T_{\nu_{\alpha}}\right), \\
& \int \cos 2 \bar{\theta}_{\mathrm{H}} f_{\bar{\nu}_{\alpha}}\left(E_{\bar{\nu}_{\alpha}}\right) d E_{\bar{\nu}_{\alpha}} \approx F_{\bar{\nu}}\left(\theta, E_{\mathrm{R}} / T_{\bar{\nu}_{\alpha}}\right),
\end{aligned}
$$

where we define the neutrino spectral integrals as,

$$
\begin{aligned}
& F_{\nu}\left(\theta, x_{\mathrm{R}}\right) \equiv \frac{1}{F_{2}(0)} \int_{0}^{\infty} \frac{1-x / x_{\mathrm{R}}}{\sqrt{\left(1-x / x_{\mathrm{R}}\right)^{2}+\tan ^{2} 2 \theta}} \frac{x^{2}}{\exp (x)+1} d x, \\
& F_{\bar{\nu}}\left(\theta, x_{\mathrm{R}}\right) \equiv \frac{1}{F_{2}(0)} \int_{0}^{\infty} \frac{1+x / x_{\mathrm{R}}}{\sqrt{\left(1+x / x_{\mathrm{R}}\right)^{2}+\tan ^{2} 2 \theta}} \frac{x^{2}}{\exp (x)+1} d x .
\end{aligned}
$$

Clearly, for $\tan 2 \theta \ll 1, F_{\bar{\nu}}\left(\theta, x_{\mathrm{R}}\right) \approx 1$. Here $E_{\mathrm{R}}$ is the energy corresponding to a neutrino at resonance at radius $r$.

With these definitions, and for small $B_{e \tau}$, we can reduce Eq. (31a) for $B$ to,

$$
\begin{aligned}
B \approx-\sqrt{2} G_{\mathrm{F}} \frac{\left[1-\sqrt{1-R_{\nu}^{2} / r^{2}}\right]^{2}}{4} & n_{\nu_{e}}^{0} F_{\nu}\left(\theta, E_{\mathrm{R}} / T_{\nu_{e}}\right)-n_{\nu_{\tau}}^{0} F_{\nu}\left(\theta, E_{\mathrm{R}} / T_{\nu_{\tau}}\right) \\
& \left.+n_{\bar{\nu}_{e}}^{0} F_{\bar{\nu}}\left(\theta, E_{\mathrm{R}} / T_{\bar{\nu}_{e}}\right)-n_{\bar{\nu}_{\tau}}^{0} F_{\bar{\nu}}\left(\theta, E_{\mathrm{R}} / T_{\bar{\nu}_{\tau}}\right)\right],
\end{aligned}
$$

where $n_{\nu_{e}}^{0}, n_{\nu_{\tau}}^{0}, n_{\bar{\nu}_{e}}^{0}$, and $n_{\bar{\nu}_{\tau}}^{0}$ are the appropriate neutrino or antineutrino number densities at the neutrino sphere as in Eq. (12b). This zero-order expression for $B$ is to be used in Step (2) in the iterative procedure outlined above. To proceed further requires that we estimate $B_{e \tau}$. 
The angular integration for Eq. (31b) is the same as for Eq. (31a). In performing the angular integration in Eq. (31b) we will again assume that $B_{e \tau}$ is small. The energy dependent integrals in Eq. (31b) can be written as:

$$
\begin{aligned}
& \int \sin 2 \theta_{\mathrm{H}} f_{\nu_{\alpha}}\left(E_{\nu_{\alpha}}\right) d E_{\nu_{\alpha}} \approx G_{\nu}\left(\theta, E_{\mathrm{R}} / T_{\nu_{\alpha}}\right), \\
& \int \sin 2 \bar{\theta}_{\mathrm{H}} f_{\bar{\nu}_{\alpha}}\left(E_{\bar{\nu}_{\alpha}}\right) d E_{\bar{\nu}_{\alpha}} \approx G_{\bar{\nu}}\left(\theta, E_{\mathrm{R}} / T_{\bar{\nu}_{\alpha}}\right) .
\end{aligned}
$$

In like manner to Eqs. (34a \& b) we define,

$$
\begin{aligned}
& G_{\nu}\left(\theta, x_{\mathrm{R}}\right) \equiv \frac{1}{F_{2}(0)} \int_{0}^{\infty} \frac{\tan 2 \theta}{\sqrt{\left(1-x / x_{\mathrm{R}}\right)^{2}+\tan ^{2} 2 \theta}} \frac{x^{2}}{\exp (x)+1} d x, \\
& G_{\bar{\nu}}\left(\theta, x_{\mathrm{R}}\right) \equiv \frac{1}{F_{2}(0)} \int_{0}^{\infty} \frac{\tan 2 \theta}{\sqrt{\left(1+x / x_{\mathrm{R}}\right)^{2}+\tan ^{2} 2 \theta}} \frac{x^{2}}{\exp (x)+1} d x,
\end{aligned}
$$

where the notation is as in Eqs. (34a \& b).

Finally, we can utilize Eqs. (36a-37b) to give an approximate expression for $B_{e \tau}$,

$$
\begin{aligned}
B_{e \tau} \approx \sqrt{2} G_{\mathrm{F}} \frac{\left[1-\sqrt{1-R_{\nu}^{2} / r^{2}}\right]^{2}}{4} & n_{\nu_{e}}^{0} G_{\nu}\left(\theta, E_{\mathrm{R}} / T_{\nu_{e}}\right)-n_{\nu_{\tau}}^{0} G_{\nu}\left(\theta, E_{\mathrm{R}} / T_{\nu_{\tau}}\right) \\
& \left.+n_{\bar{\nu}_{e}}^{0} G_{\bar{\nu}}\left(\theta, E_{\mathrm{R}} / T_{\bar{\nu}_{e}}\right)-n_{\bar{\nu}_{\tau}}^{0} G_{\bar{\nu}}\left(\theta, E_{\mathrm{R}} / T_{\bar{\nu}_{\tau}}\right)\right] .
\end{aligned}
$$

The notation in this equation is the same as in Eq. (35). The approximations for $B$ and $B_{e \tau}$ in Eqs. (35) and (38), respectively, are valid when, $\left|B_{e \tau}\right| /\left(\delta m^{2} / 2 E_{\mathrm{R}}\right) \sin 2 \theta \ll 1$.

Note that the integrand in the expression for $G_{\nu}\left(\theta, x_{\mathrm{R}}\right)$ in Eq. (37a) contains a factor, $\sin \theta_{\mathrm{H}} \approx \tan 2 \theta / \sqrt{\left(1-x / x_{\mathrm{R}}\right)^{2}+\tan ^{2} 2 \theta}=\tan 2 \theta / \sqrt{\left(1-E_{\nu} / E_{\mathrm{R}}\right)^{2}+\tan ^{2} 2 \theta}$, which is sharply peaked at $E_{\nu}=E_{\mathrm{R}}$ for small vacuum mixing angles. In Fig. 5 we plot $\sin 2 \theta_{\mathrm{H}}$ as a function of $E_{\nu} / E_{\mathrm{R}}$ for three values of the vacuum mixing angle. The dotted line in this figure corresponds to $\tan 2 \theta=10^{-3}$. The dashed line corresponds to $\tan 2 \theta=10^{-2}$, while 
the solid line is for $\tan 2 \theta=0.1$. Since the factor $\sin 2 \theta_{\mathrm{H}}$ appears in the integration over the neutrino energy spectrum we can see easily that the smaller the vacuum mixing angle, the smaller will be the fraction of the total number density of neutrinos which contribute to $B_{e \tau}$. The physical interpretation of this is clear: the neutrinos which make the largest contribution to the off-diagonal neutrino background terms are those which have the largest full effective mixing angles at the position under consideration at radius $r$. These are the neutrinos which have energies close to $E_{\mathrm{R}}$.

With the iterative procedure outlined above we can estimate $B$ and $B_{e \tau}$ for adiabatic neutrino flavor evolution in both the shock re-heating epoch and the hot bubble/ $r$-process nucleosynthesis epoch. Refs. [5] and [6] give detailed expositions of the expected neutrino emission parameters for these epochs. Typical neutrino luminosities for the shock reheating epoch at $t_{\mathrm{PB}} \approx 0.15 \mathrm{~s}$ (see the discussion in Ref. [5]) are $L_{\nu_{e}} \approx L_{\bar{\nu}_{e}} \approx L_{\nu_{\tau(\mu)}} \approx$ $L_{\bar{\nu}_{\tau(\mu)}} \approx 5 \times 10^{52} \mathrm{erg} \mathrm{s}^{-1}$. The neutrino sphere radius at this epoch is $R_{\nu} \approx 50 \mathrm{~km}$, while the average neutrino energies are $\left\langle E_{\nu_{e}}\right\rangle \approx 9 \mathrm{MeV},\left\langle E_{\bar{\nu}_{e}}\right\rangle \approx 12 \mathrm{MeV}$, and $\left\langle E_{\nu_{\tau(\mu)}}\right\rangle \approx$ $\left\langle E_{\bar{\nu}_{\tau(\mu)}}\right\rangle \approx 20 \mathrm{MeV}$. By contrast, in the later hot bubble/r-process nucleosynthesis epoch $\left(t_{\mathrm{PB}} \approx 5 \mathrm{~s}\right)$ the neutrino liminosities are $L_{\nu_{e}} \approx L_{\bar{\nu}_{e}} \approx L_{\nu_{\tau(\mu)}} \approx L_{\bar{\nu}_{\tau(\mu)}} \approx 10^{51} \mathrm{erg} \mathrm{s}^{-1}$, while the neutrino sphere is at radius $R_{\nu} \approx 10 \mathrm{~km}$. The average neutrino energies for this epoch are $\left\langle E_{\nu_{e}}\right\rangle \approx 11 \mathrm{MeV},\left\langle E_{\bar{\nu}_{e}}\right\rangle \approx 16 \mathrm{MeV}$, and $\left\langle E_{\nu_{\tau(\mu)}}\right\rangle \approx\left\langle E_{\bar{\nu}_{\tau(\mu)}}\right\rangle \approx 25 \mathrm{MeV}$.

As Ref. [5] shows, for a substantial enhancement in shock re-heating $\nu_{\tau}$ (or $\nu_{\mu}$ ) neutrinos with energies $E_{\nu} \approx 35 \mathrm{MeV}$ must be efficiently transformed into $\nu_{e}$ neutrinos in the region behind the stalled shock. Ref. [6] shows that neutrinos with energies $E_{\nu} \approx 25$ 
$\mathrm{MeV}$ are the most important in determining the electron fraction, $Y_{e}$, in the hot bubble $/ r$ process nucleosynthesis epoch.

For shock re-heating enhancement we must have adiabatic transformation of neutrinos with energies $E_{\nu} \approx 35 \mathrm{MeV}$. In the hot bubble $/ r$-process nucleosynthesis epoch adiabatic transformation is not necessary to drive the material too proton rich for $r$-process nucleosynthesis to occur $\left(Y_{e}>0.5\right)$. In fact Ref. [6] shows that $\nu_{\tau(\mu)} \rightleftharpoons \nu_{e}$ flavor conversion efficiencies as small as $\sim 30 \%$ for neutrinos with energies $E_{\nu} \approx 25 \mathrm{MeV}$ will suffice to drive $Y_{e} \geq 0.5$. Nevertheless, for large enough vacuum mixing angles, adiabatic transformation of neutrinos with $E_{\nu} \approx 25 \mathrm{MeV}$ will occur in some regions of the $\left(\delta m^{2}, \sin ^{2} 2 \theta\right)$ plot (Fig. 2 in Ref. [6]).

Consider adiabatic neutrino flavor conversion specifically for $E_{\nu}=35 \mathrm{MeV}$ in the shock re-heating epoch and $E_{\nu}=25 \mathrm{MeV}$ in the hot bubble/r-process nucleosynthesis epoch. For comparison, we first present values of $\delta m^{2}$ and $\sin ^{2} 2 \theta$ which give an adiabaticity parameter $\gamma=3$ for the bare electron number density distributions relevant for these epochs. In Figs. 6 and 7, the solid contour lines for $\gamma=3$ correspond to these values of $\delta m^{2}$ and $\sin ^{2} 2 \theta$ for the representative conditions in the shock re-heating and hot bubble/rprocess nucleosynthesis epochs, respectively.

It should be noted that the adiabatic approximation will be valid over the whole range of neutrino energies implicit in the neutrino distribution functions entering into the expressions for $B$ and $B_{e \tau}$. Neutrinos with energies $E_{\nu}<E_{\mathrm{R}}$ will propagate through resonances prior to reaching the resonant position for the specific example neutrino energy under discussion (either $E_{\mathrm{R}}=35 \mathrm{MeV}$ or $E_{\mathrm{R}}=25 \mathrm{MeV}$ ). It is a general feature of the 
density scale height above the neutrino sphere that the neutrinos with energies $E_{\nu}<E_{\mathrm{R}}$ will experience adiabatic flavor evolution through their resonances as long as neutrinos with energy $E_{\mathrm{R}}$ go through the resonance adiabatically $[5,6]$. Background neutrinos with energies $E_{\nu}>E_{\mathrm{R}}$ will not have gone through resonances and therefore evolve adiabatically prior to arriving at the resonance position for a neutrino with energy $E_{\mathrm{R}}$. We conclude that the expressions for $B$ and $B_{e \tau}$ in Eqs. (31a \& b) are appropriate for the example under consideration.

Using the iterative procedure outlined above we can calculate the true adiabatic parameter, $\gamma\left(t_{\text {res }}\right)$, including the neutrino background contributions. We show the new contour lines for $\gamma=3$ as dotted lines in Figs. 6 and 7 for the respective epochs. We can easily see that the neutrino background has a completely negligible effect on adiabaticity at resonance along the solid $\gamma=3$ contour line in Fig. 6. The new contour line for $\gamma=3$ in Fig. 6 is indistinguishable from the one calculated for the bare electron number density. The new contour line for $\gamma=3$ in Fig. 7 moves a little bit to the right of the solid line, but the neutrino background effects are also evidently small.

Any neutrino mixing parameters $\delta m^{2}$ and $\sin ^{2} 2 \theta$ which are to the right of the $\gamma=3$ contour lines in Figs. 6 and 7 correspond to larger values of $\gamma$ for the specific example neutrino energies under discussion. For a given $\delta m^{2}$ the ratio $\left|B_{e \tau}\right| /\left(\delta m^{2} / 2 E_{\mathrm{R}}\right) \sin 2 \theta$ will decrease as $\sin ^{2} 2 \theta$ and, hence, $\gamma$ increases. The off-diagonal neutrino background contribution will have a negligible effect on neutrino flavor conversion everywhere to the right of the contour lines in Figs. 6 and 7. Likewise, $B$ is roughly constant for a given $\delta m^{2}$ as $\sin ^{2} 2 \theta$ and, hence, $\gamma$ is increased. The diagonal contribution of the neutrino background 
produces a negligible alteration in the computed flavor conversion efficiencies everywhere to the right of the contour lines in Figs. 6 and 7.

We have also examined adiabatic neutrino flavor conversion in supernovae for a range of neutrino energies. We can conclude that the neutrino background, specifically $B$ and $B_{e \tau}$, will not result in any modification of the results of Refs. [5] and [6] whenever adiabatic neutrino flavor evolution is at issue.

\section{IIIb.) Nonadiabatic Neutrino Flavor Evolution}

The effects of the neutrino background on nonadiabatic neutrino flavor evolution in the region above the neutrino sphere are potentially more significant than are the neutrino background effects on adiabatic neutrino flavor evolution. In general, the evaluation of $B$ and $B_{e \tau}$ from Eqs. (22a \& b) is considerably more complicated when neutrino flavor evolution is nonadiabatic than it is when the adiabatic limit for neutrino flavor evolution obtains.

A neutrino of energy $E_{\mathrm{R}}$, nonadiabatically going through a resonance at a point above the neutrino sphere, experiences a neutrino background effect which depends on the prior histories of all the neutrinos in the ensemble which are passing through the resonance region. In this case, we cannot argue that background neutrinos with $E_{\nu}<E_{\mathrm{R}}$ go through resonances adiabatically. The flavor evolution for background neutrinos with $E_{\nu}>E_{\mathrm{R}}$ can still be considered adiabatic for the purposes of calculating $B$ and $B_{e \tau}$, since these neutrinos will not yet have gone through resonances when they are in the resonance region for energy $E_{\mathrm{R}}$. 
In Fig. 8 we graphically illustrate the difficulties inherent in computing $B$ and $B_{e \tau}$ from Eqs. (22a \& b) for nonadiabatic neutrino flavor evolution. In this figure we show the radial path of a neutrino with energy $E_{\mathrm{R}}$. The resonance position for this neutrino is the point labeled $\operatorname{RES}\left(E_{\mathrm{R}}\right)$. The path for a neutrino of energy $E_{\mathrm{B}}$ representative of the neutrino background at the point $\operatorname{RES}\left(E_{\mathrm{R}}\right)$ is labeled by $E_{\mathrm{B}}$. If $E_{\mathrm{B}}<E_{\mathrm{R}}$ then the neutrino on the path labeled by $E_{\mathrm{B}}$ presumably propagated through a resonance of its own prior to reaching position $\operatorname{RES}\left(E_{\mathrm{R}}\right)$. The resonace position for the background neutrino is labeled $\operatorname{RES}\left(E_{\mathrm{B}}\right)$. Whether or not this background neutrino experiences flavor conversion at $\operatorname{RES}\left(E_{\mathrm{B}}\right)$ depends, in turn, on the flavor evolution histories of the background neutrinos which pass through this point. The paths for some of these "secondary" background neutrinos are shown in Fig. 8.

As we can see from Fig. 8, an exact calculation of the neutrino background contributions requires us to simultaneously follow the flavor evolution histories of neutrinos with different energies on all possible neutrino paths above the neutrino sphere. This could be done in a Monte Carlo calculation. However, there is a simpler alternative if we make note of the following two facts. First, we are most interested in regions which are far away from the neutrino sphere. The region for $r$-process nucleosynthesis in the hot bubble is located at radii $r>4 R_{\nu}$. So the polar angles for neutrino paths to a point in this region lie in a narrow range around $\theta_{\mathbf{q}}=0$. In addition, at a point close to the neutrino sphere where the polar angles for the relevant neutrino paths can be significantly different from zero, the electron number density is so high that neutrino background effects can be safely ignored. Therefore, we can make an approximation and take the flavor evolution history of 
a radially propagating neutrino $\left(\theta_{\mathbf{q}}=0\right)$ as representative of the flavor evolution histories of all neutrinos with the same energy.

The flavor evolution history of radially propagating neutrinos for a given set of $\delta m^{2}$ and $\sin ^{2} 2 \theta$ can then be calculated with the following procedure:

$\left(1^{\prime}\right.$.) We numerically represent the neutrino energy spectrum with a grid of energy bins. These energy bins cover a neutrino energy range of 1-100 MeV. Typically our numerical calculations employ $\sim 200$ energy bins. Since neutrinos with lower energies go through resonances first, we start the calculations at the lower end of the energy grid.

$\left(2^{\prime}\right.$.) For the particular grid point (neutrino energy bin) at neutrino energy $E_{\nu}$, we use the iterative procedure outlined at the beginning of this section to locate the resonance position, $t_{\mathrm{res}}\left(E_{\nu}\right)$, for this particular neutrino energy $E_{\nu}$. As a byproduct of this iterative procedure, we will obtain the corresponding neutrino background contributions $B$ and $B_{e \tau}$ at this position $t_{\mathrm{res}}\left(E_{\nu}\right)$. The evaluation of $B$ and $B_{e \tau}$ in this case is quite similar to that for the case of adiabatic neutrino flavor evolution, except that here we must use Eqs. (22a \& b) together with the flavor evolution histories of neutrinos with energies lower than $E_{\nu}$.

$\left(3^{\prime}.\right)$ Using the resonance position, $t_{\mathrm{res}}\left(E_{\nu}\right)$, and the corresponding neutrino background contributions $B$ and $B_{e \tau}$ from Step $\left(2^{\prime}\right)$, we can evaluate the Landau-Zener probability $P_{\mathrm{LZ}}\left(E_{\nu}\right)$ (Eq. [28]) for a neutrino with energy $E_{\nu}$ to jump from one mass eigenstate to the other in the course of transversing the resonance region.

(4'.) The flavor evolution history of $\nu_{e}$ neutrinos with energy $E_{\nu}$ is then approximated as

$$
\left|a_{1 e}(t)\right|^{2} \approx \begin{cases}0 & \text { if } t \leq t_{\mathrm{res}}\left(E_{\nu}\right) \\ P_{\mathrm{LZ}}\left(E_{\nu}\right) & \text { otherwise. }\end{cases}
$$


Likewise, the flavor evolution history of $\nu_{\tau}$ neutrinos with energy $E_{\nu}$ is approximated as

$$
\left|a_{1 \tau}(t)\right|^{2} \approx \begin{cases}1 & \text { if } t \leq t_{\mathrm{res}}\left(E_{\nu}\right) \\ 1-P_{\mathrm{LZ}}\left(E_{\nu}\right) & \text { otherwise }\end{cases}
$$

In the above two equations, the evolutionary parameter $t$ increases away from the neutrino sphere. These approximations for the neutrino flavor evolution history, together with Eqs. $(22 \mathrm{a} \& \mathrm{~b})$, are then used in the iterative procedure in Step $\left(2^{\prime}\right)$ to locate the resonance position and calculate the corresponding neutrino background contributions for neutrinos with energies higher than $E_{\nu}$.

At the end of the above procedure, we will have obtained the approximate flavor evolution histories for all the neutrino energies on the energy grid. This information then can be used to calculate the electron fraction $Y_{e}$ in the $r$-process nucleosynthesis region as described in Ref. [6]. We present the new $Y_{e}=0.5$ line, including the neutrino background effects, as a dotted contour line on the $\left(\delta m^{2}, \sin ^{2} 2 \theta\right)$ plot in Fig. 9. The original $Y_{e}=0.5$ line in Fig. 2 of Ref. [6] is shown as the solid contour line in Fig. 9. To the right of the $Y_{e}=0.5$ line, the material will be driven too proton rich for $r$-process nucleosynthesis to occur in the hot bubble.

By examining the two contour lines in Fig. 9, we can draw two conclusions. First, with a proper treatment of the neutrino background effects, we see that $r$-process nucleosynthesis in the hot bubble remains a sensitive probe of the flavor-mixing properties of neutrinos with cosmologically significant masses. In fact, inclusion of the neutrino background contributions results in a small modification of the original $Y_{e}=0.5$ line for $\delta m^{2}=4 \mathrm{eV}^{2}$ to $\delta m^{2}=10^{4} \mathrm{eV}^{2}$. Furthermore, after we take into account the neutrino background contributions, it is evident that the range of neutrino vacuum mass-squared difference $\delta m^{2}$ 
probed by $r$-process nucleosynthesis is extended down to $\delta m^{2}<2 \mathrm{eV}^{2}$. The reason for this extension can be found in the nonlinear nature of neutrino flavor transformation in the presence of a neutrino background.

Close to the neutrino sphere where little neutrino flavor transformation has occurred, the number density of $\nu_{e}$ neutrinos is larger than that of $\nu_{\tau}$ neutrinos. This is because the luminosities for $\nu_{e}$ and $\nu_{\tau}$ are approximately the same, but the average $\nu_{\tau}$ neutrino energy is much higher (cf. Eq. [12b]). However, with neutrino flavor transformation, more $\nu_{e}$ neutrinos are transformed into $\nu_{\tau}$ neutrinos than $\nu_{\tau}$ neutrinos are transformed into $\nu_{e}$ neutrinos. This is because there are more low energy $\nu_{e}$ neutrinos and only low energy neutrinos are very efficiently transformed for the parameters along the dotted contour line in Fig. 9. Because of the nonlinear evolution of the neutrino background, the diagonal contribution $B$ evolves from a positive value for positions close to the neutrino sphere to a negative value for positions far away from the neutrino sphere. Neutrinos with $\delta m^{2}<2$ $\mathrm{eV}^{2}$ and energies over a broad range will tend to have resonances far enough out that the diagonal contributions will satisfy $B<0$. For a given $\delta m^{2}$ and a given energy $E_{\nu}$, the resonance position will lie closer to the neutrino sphere for the case $B<0$ than it would for the case where no neutrino background is present (cf. Eq. [26]).

As Ref. [6] discusses, $Y_{e}$ and, hence, $r$-process nucleosynthesis are sensitive to neutrino flavor conversion only when resonances occur inside the weak freeze-out radius. The weak freeze-out radius is the radius beyond which typical $\nu_{e}$ and $\bar{\nu}_{e}$ capture rates are small compared to the material expansion rate. When $B<0$, the resonances for given $\delta m^{2}$ are 
drawn in toward the neutrino sphere. Hence, we find that the $Y_{e}=0.5$ line drops to lower values of $\delta m^{2}$ in the presence of a neutrino background.

\section{Conclusions}

We have calculated neutrino flavor transformation in the region above the neutrino sphere in Type II supernovae including all contributions from the neutrino background. In particular, we have examined the neutrino background effects on both cases of adiabatic and nonadiabatic neutrino flavor evolution. In the case of adiabatic neutrino flavor evolution, which is most relevant for supernova shock re-heating, we find that the neutrino background has a completely negligible effect on the range of vacuum mass-squared difference, $\delta m^{2}$, and vacuum mixing angle, $\theta$, or equivalently $\sin ^{2} 2 \theta$, required for enhanced shock heating. In the case of nonadiabatic neutrino flavor evolution relevant for $r$-process nucleosynthesis in the hot bubble, we find that $r$-process nucleosynthesis from neutrinoheated supernova ejecta remains a sensitive probe of the mixing between a light $\nu_{e}$ and a $\nu_{\tau(\mu)}$ with a cosmologically significant mass. The modification of the $\left(\delta m^{2}, \sin ^{2} 2 \theta\right)$ parameter region probed by $r$-process nucleosynthesis due to the neutrino background effects is generally small. The nonlinear nature of neutrino flavor transformation in the presence of a neutrino background actually extends the sensitivity of $r$-process nucleosynthesis to smaller values of $\delta m^{2}$.

In general, we find that a proper account of neutrino background effects leads to no modification in the overall qualitative conclusions of Refs. [5] and [6]. At the early

epochs of the post-core-bounce supernova environment $\left(t_{\mathrm{PB}}<1 \mathrm{~s}\right)$, we find that the 
characteristically large electron number densities and large density scale heights determine the phenomenon of neutrino flavor transformation. Even at the later epochs associated with $r$-process nucleosynthesis, the effects of the neutrino background on neutrino flavor evolution are small.

\section{Acknowledgments}

We want to thank J. R. Wilson and R. W. Mayle for much patient education on the subject of supernova neutrinos. We would like to acknowledge discussions with A. B. Balantekin and W. C. Haxton. Y.-Z. Qian also acknowledges discussions with M. Herrmann and M. Burkardt. This work was supported by the Department of Energy under Grant No. DE-FG06-90ER40561 at the Institute for Nuclear Theory and by NSF Grant No. PHY-9121623 at UCSD. 


\section{References}

[1] G. Sigl and G. Raffelt, Nucl. Phys. B406, 423 (1993).

[2] S. Samuel, Phys. Rev. D 48, 1462 (1993).

[3] G. M. Fuller, R. W. Mayle, J. R. Wilson, and D. N. Schramm, Astrophys. J. 322, 795 (1987).

[4] D. Nötzold and G. Raffelt, Nucl. Phys. B307, 924 (1988).

[5] G. M. Fuller, R. W. Mayle, B. S. Meyer, and J. R. Wilson, Astrophys. J. 389, 517 (1992).

[6] Y.-Z. Qian, G. M. Fuller, G. J. Mathews, R. W. Mayle, J. R. Wilson, and S. E. Woosley, Phys. Rev. Lett. 71, 1965 (1993).

[7] L. Wolfenstein, Phys. Rev. D 17, 2369 (1978); 20, 2634 (1979); S. P. Mikheyev and A. Yu. Smirnov, Nuovo Cimento Soc. Ital. Fis. 9C, 17 (1986); H. A. Bethe, Phys. Rev. Lett. 56, 1305 (1986).

[8] W. C. Haxton, Phys. Rev. D 36, 2283 (1987). 


\section{Figure Captions}

Fig. 1 A generic Feynman graph for $\nu_{e}-e$ scattering.

Fig. 2 The geometrical arrangement of a neutrino sphere with radius $R_{\nu}$, a point above the neutrino sphere at radius $r$, and various neutrino paths.

Fig. 3 Generic Feynman graphs for neutrino-neutrino exchange-scattering processes. Fig. 3a is for $\nu_{e}-\nu_{e}$ scattering and Fig. $3 \mathrm{~b}$ is for $\nu_{\tau}-\nu_{\tau}$ scattering.

Fig. 4 Graphic representation for off-diagonal contributions from the neutrino background.

Fig. 5 The zero-order expression for $\sin 2 \theta_{\mathrm{H}}$ as a function of $E_{\nu} / E_{\mathrm{R}}$ for three different vacuum mixing angles. The dotted line corresponds to $\tan 2 \theta=10^{-3}$. The dashed line corresponds to $\tan 2 \theta=10^{-2}$, while the solid line is for $\tan 2 \theta=0.1$.

Fig. 6 Contour lines for $\gamma=3$ on the $\left(\delta m^{2}, \sin ^{2} 2 \theta\right)$ plot for the shock re-heating epoch. The solid contour line is calculated for the bare electron number density. The dotted line, which cannot be distinguished from the solid line in this case, is calculated with the neutrino background contributions.

Fig. 7 As in Fig. 6, but for the hot bubble/r-process nucleosynthesis epoch.

Fig. 8 Illustration of the difficulties inherent in computing the neutrino background contributions $B$ and $B_{e \tau}$ for the case of nonadiabatic neutrino flavor evolution. The radial path of a neutrino with energy $E_{\mathrm{R}}$ and resonance position $\operatorname{RES}\left(E_{\mathrm{R}}\right)$ is shown. The path for a neutrino of energy $E_{\mathrm{B}}$ representative of the neutrino background at position $\operatorname{RES}\left(E_{\mathrm{R}}\right)$ is shown together with its resonance position $\operatorname{RES}\left(E_{\mathrm{B}}\right)$. Paths for background neutrinos at position $\operatorname{RES}\left(E_{\mathrm{B}}\right)$ are also shown. 
Fig. 9 Contour lines for $Y_{e}=0.5$ are shown on the $\left(\delta m^{2}, \sin ^{2} 2 \theta\right)$ plot. The solid line is the same as the $Y_{e}=0.5$ line in Fig. 2 of Ref. [6], whereas the dotted line is calculated with the full neutrino background contributions. 
This figure "fig1-1.png" is available in "png" format from: http://arxiv.org/ps/astro-ph/9406073v1 
This figure "fig2-1.png" is available in "png" format from: http://arxiv.org/ps/astro-ph/9406073v1 
This figure "fig3-1.png" is available in "png" format from: http://arxiv.org/ps/astro-ph/9406073v1 
This figure "fig4-1.png" is available in "png" format from: http://arxiv.org/ps/astro-ph/9406073v1 
This figure "fig5-1.png" is available in "png" format from: http://arxiv.org/ps/astro-ph/9406073v1 
This figure "fig1-2.png" is available in "png" format from: http://arxiv.org/ps/astro-ph/9406073v1 
This figure "fig1-3.png" is available in "png" format from: http://arxiv.org/ps/astro-ph/9406073v1 
This figure "fig1-4.png" is available in "png" format from: http://arxiv.org/ps/astro-ph/9406073v1 
This figure "fig1-5.png" is available in "png" format from: http://arxiv.org/ps/astro-ph/9406073v1 


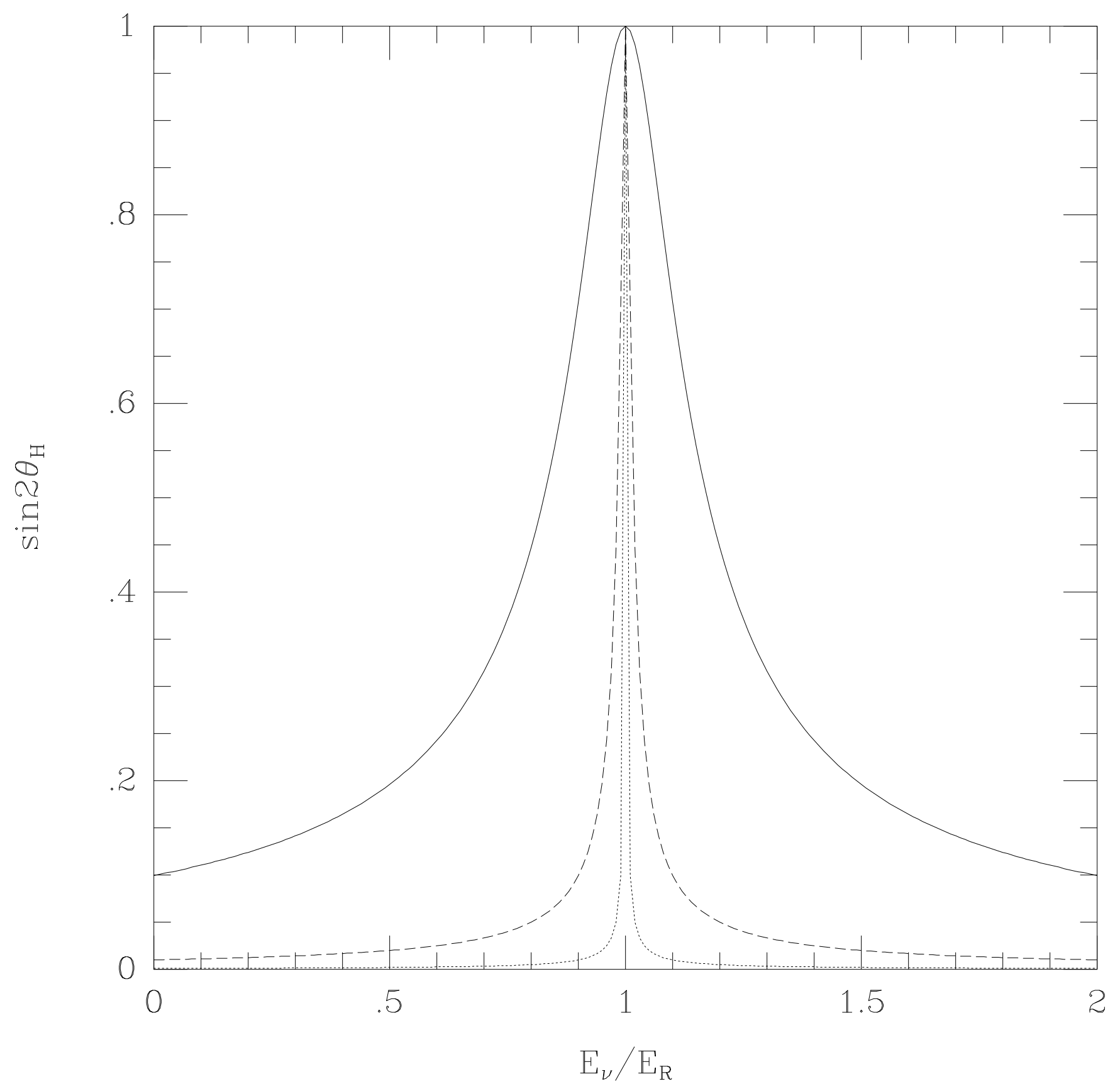

Fig. 5 


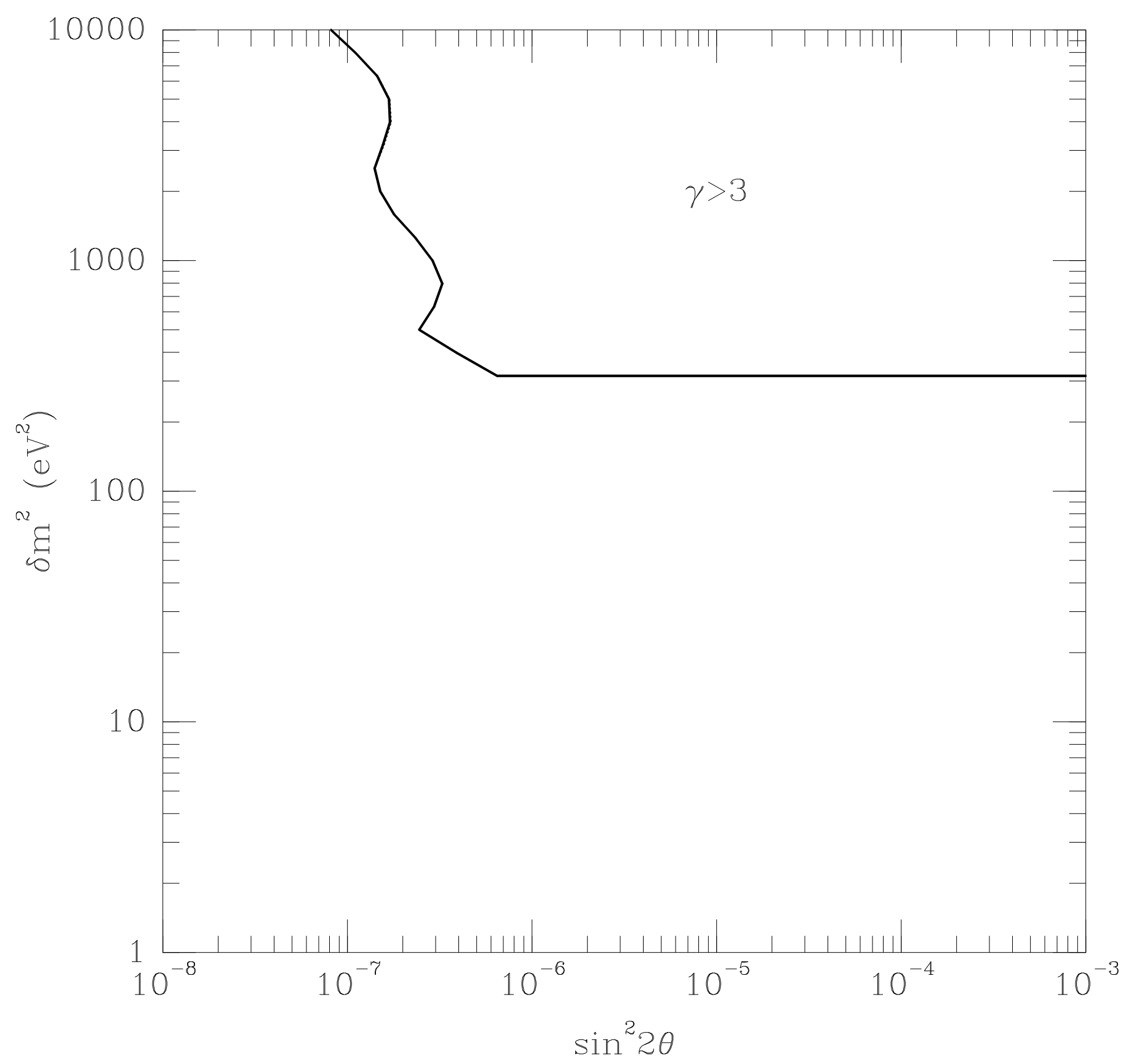

Fig. 6 


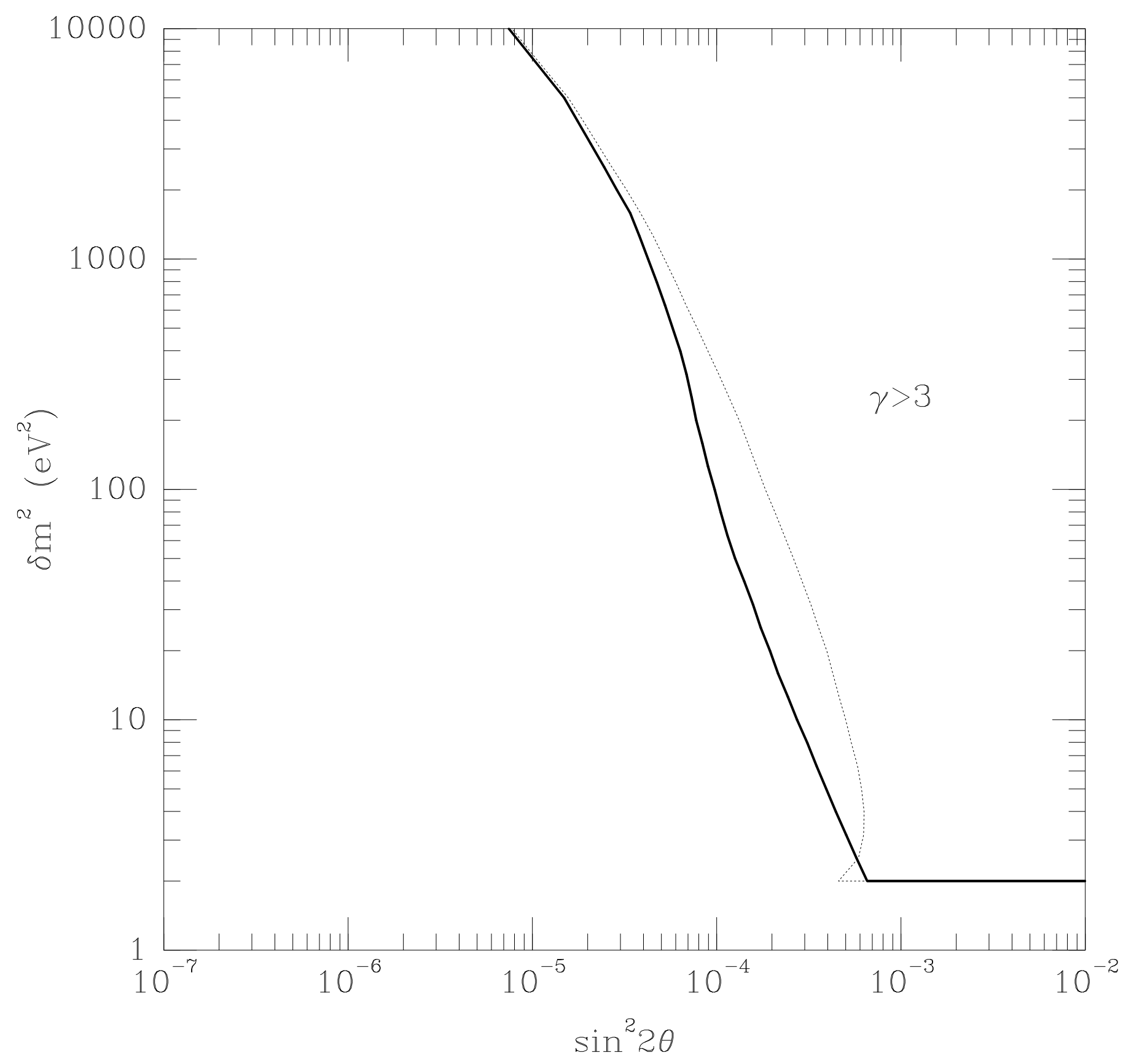

Fig. 7 


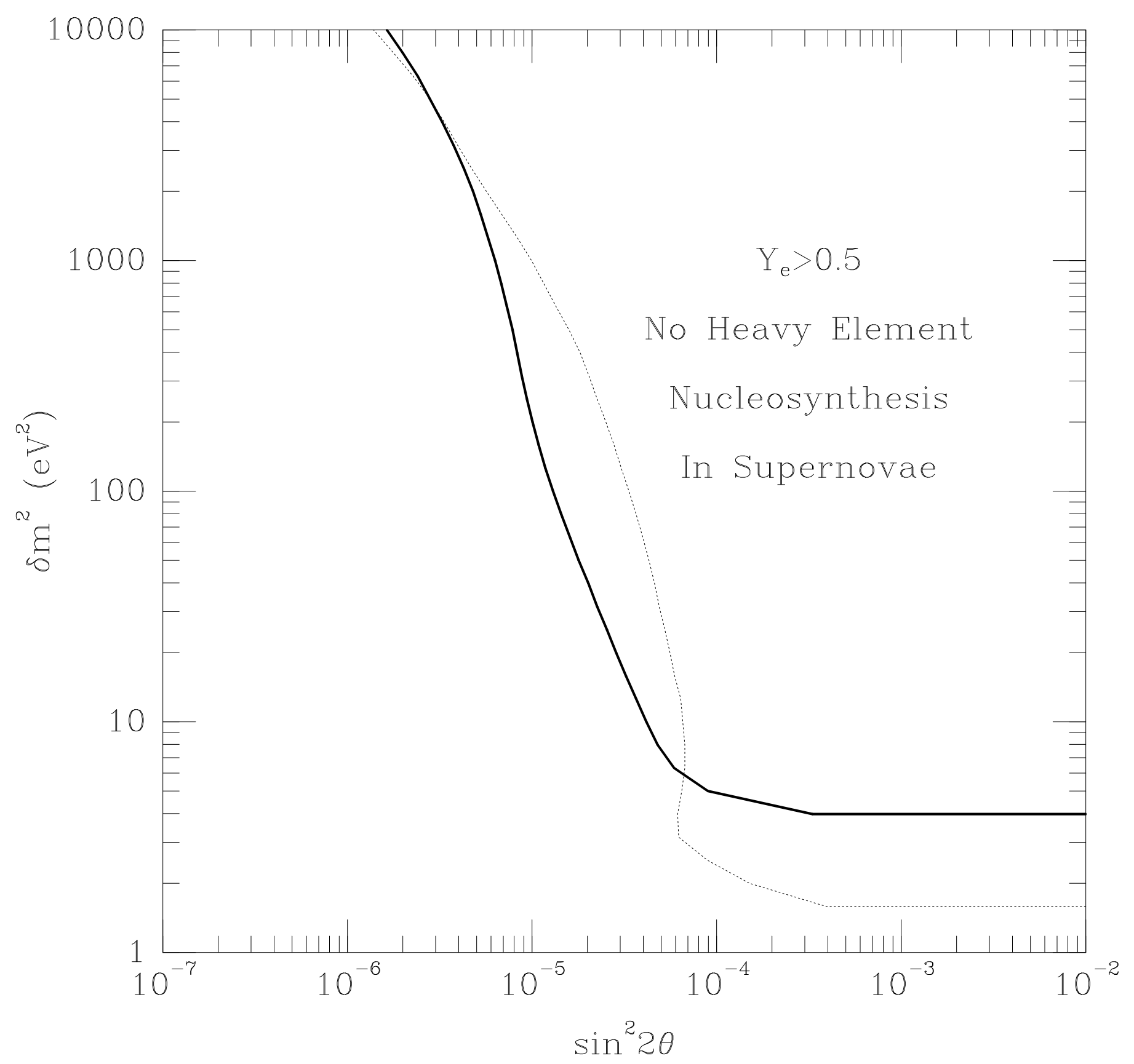

Fig. 9 


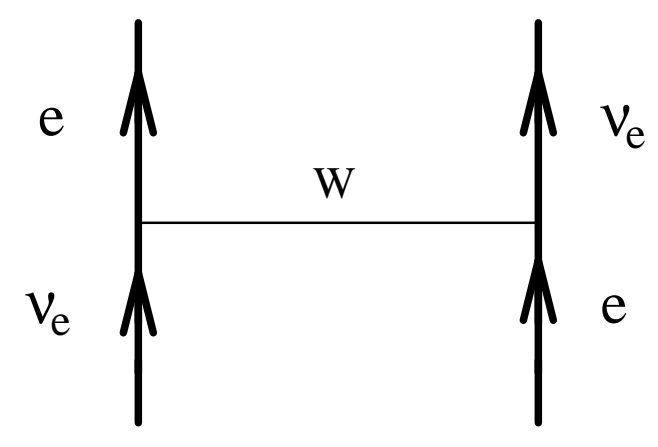

Fig. 1 


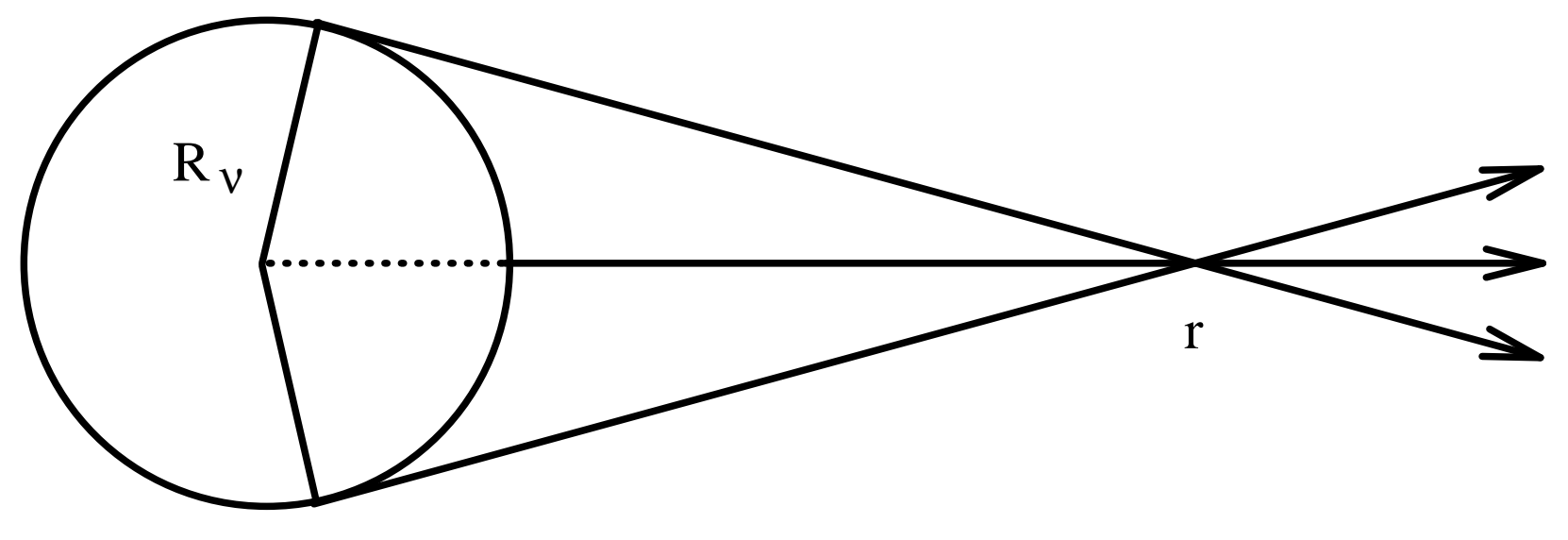

Fig. 2 


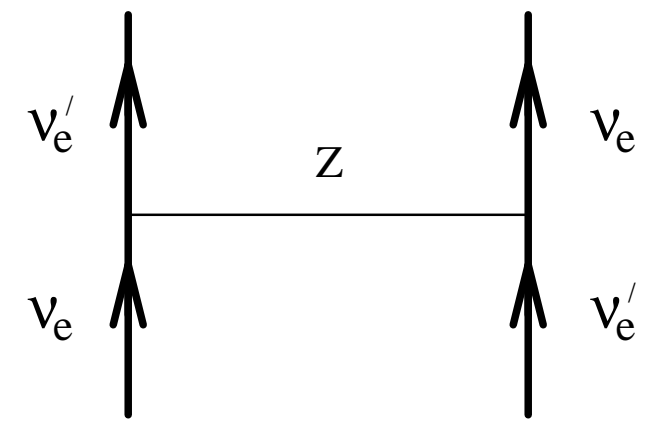

Fig. 3a

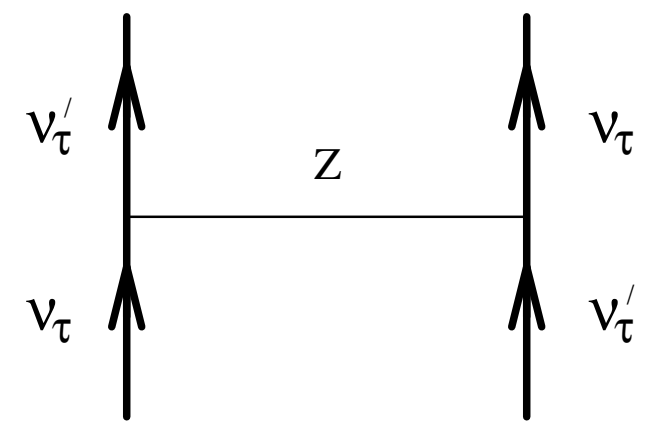

Fig. 3b 


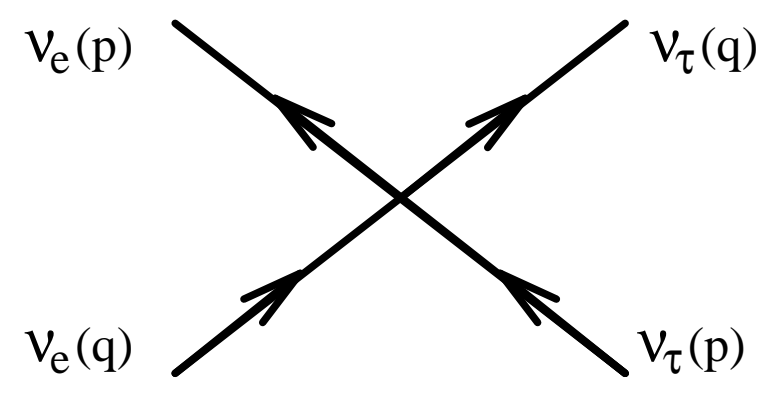

Fig. 4 


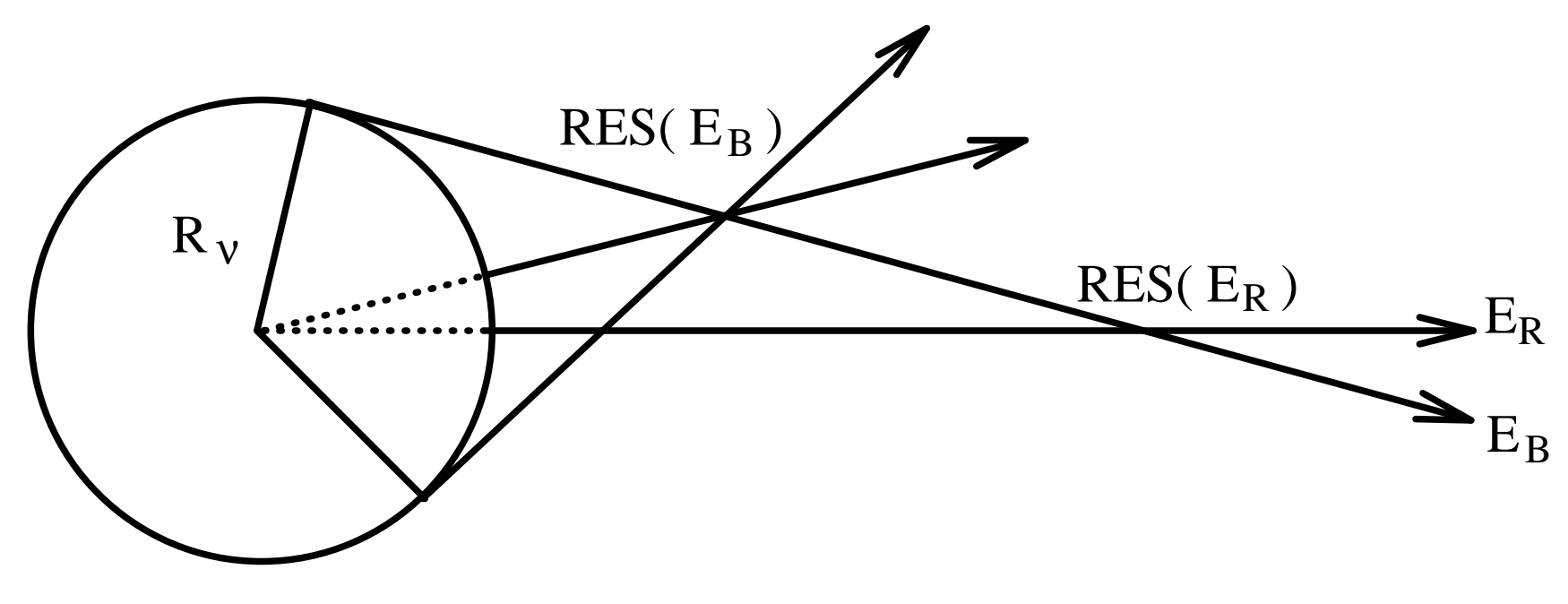

Fig. 8 